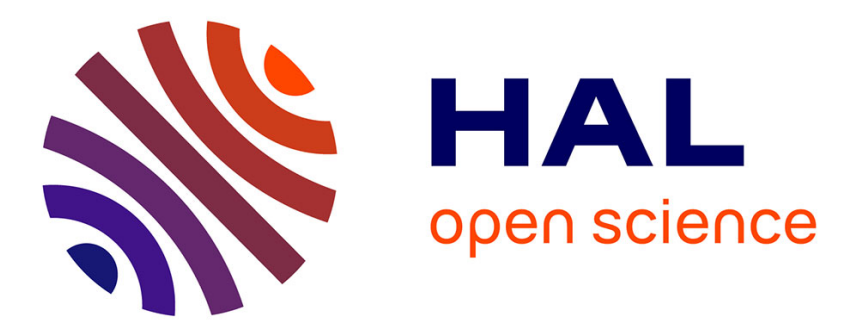

\title{
Oil Prices, Stock Markets and Portfolio Investment: Evidence from Sector Analysis in Europe over the Last Decade
}

\author{
Mohamed El Hedi Arouri, Duc Khuong Nguyen
}

\section{To cite this version:}

Mohamed El Hedi Arouri, Duc Khuong Nguyen. Oil Prices, Stock Markets and Portfolio Investment: Evidence from Sector Analysis in Europe over the Last Decade. 2010. hal-00507823

\section{HAL Id: hal-00507823 \\ https://hal.science/hal-00507823}

Preprint submitted on 1 Aug 2010

HAL is a multi-disciplinary open access archive for the deposit and dissemination of scientific research documents, whether they are published or not. The documents may come from teaching and research institutions in France or abroad, or from public or private research centers.
L'archive ouverte pluridisciplinaire HAL, est destinée au dépôt et à la diffusion de documents scientifiques de niveau recherche, publiés ou non, émanant des établissements d'enseignement et de recherche français ou étrangers, des laboratoires publics ou privés. 


\title{
Oil Prices, Stock Markets and Portfolio Investment: Evidence from Sector Analysis in Europe over the Last Decade*
}

\author{
Mohamed El Hedi Arouri \\ LEO, Université d' Orléans and EDHEC Business School, mohamed.arouri@univ-orleans.fr \\ Rue de Blois - BP 6739, 45067 Orléans cedex 2, France \\ Phone: +33238492410-Fax: +33238417380 \\ Duc Khuong Nguyen \\ ISC Paris School of Management, France,dnguyen@groupeisc.com \\ 22, Boulevard du Fort de Vaux, 75017 Paris, France \\ Phone: +33140539999-Fax: +33140539898
}

\begin{abstract}
This article extends the understanding of oil-stock market relationships over the last turbulent decade. Unlike previous empirical investigations, which have largely focused on broad-based market indices (national and/or regional indices), we examine short-term linkages in the aggregate as well as sector by sector levels in Europe using different econometric techniques. Our main findings suggest that the reactions of stock returns to oil price changes differ greatly depending on the activity sector. In the out-of-sample analysis we show that introducing oil asset into a diversified portfolio of stocks allows to significantly improve its risk-return characteristics.
\end{abstract}

Keywords: oil prices, portfolio management, short-term analysis, sector indices.

JEL classifications: G12, F3, Q43.

\footnotetext{
* The authors thank anonymous reviewers and Stéphane Grégoir for helpful comments and suggestions on an earlier version of the paper. The usual disclaimer applies.
} 


\section{Introduction}

Understanding the dynamics of stock returns is an issue of ongoing research in financial market literature. In particular, identifying the factors that drive stock market returns is of utmost relevance and importance to investors and policy makers. Although an abundance of theoretical and empirical works focused on asset pricing, there is no consensus about both the nature and number of factors of stock returns. Furthermore, as oil price has changed with sequences of very large increases and decreases over recent years, it is now quite opportune to augment the existing research on its impacts on stock market returns. Various transmission channels exist through which oil price fluctuations may affect stock returns. Indeed, the value of stock in theory equals discounted sum of expected future cash-flows. These discounted cash-flows reflect economic conditions (e.g., inflation, interest rates, production costs, income, economic growth, and investor and consumer confidence) and macroeconomic events that are likely to be influenced by oil shocks. Accordingly, oil price changes may impact stock returns.

In the literature, there has been a large volume of works on the linkages between oil prices and economic variables. The majority of these studies have shown significant effects of oil price fluctuations on economic activity for several developed and emerging countries (Hamilton, 2003; Cunado and Perez de Garcia, 2005; Balaz and Londarev, 2006; Lardic and Mignon, 2008; Gronwald, 2008; Cologni and Manera, 2008; and Kilian, 2008). By contrast, there have been relatively a few attempts to study the dynamic relationship between oil price variations and stock markets.

The pioneering paper by Jones and Kaul (1996) tests the reaction of stock returns in four developed markets (Canada, Japan, the UK, and the US) to oil price fluctuations on the basis of the standard cash-flow dividend valuation model. They find that for the US and Canada stock market reaction can be accounted for entirely by the impact of oil shocks on cash flows. The results for Japan and the UK were nevertheless inconclusive. Using an unrestricted vector autoregressive (VAR) model, Huang et al. (1996) find no evidence of a relationship between oil prices and the S\&P500 market index. Inversely, Sadorsky (1999) also applies an unrestricted VAR model with GARCH effects to American monthly data and shows a significant relationship between oil price changes and US aggregate stock returns. Park and Ratti (2008) show that oil price increases have a negative impact on stock returns in the US and twelve European countries, whereas stock markets in Norway, an oil-exporting country respond positively to rises of oil price. In a more recent study, Apergis and Miller (2009) also examine whether structural oil-market shocks affect stock returns in eight developed countries, 
and document no significant responses of international stock market returns to oil price shocks.

Very few studies have looked at the impact of oil price changes on the stocks of individual sectors. In addition, most of these studies are country-specific and therefore do not provide a global perspective. For instance, Sadorsky (2001) and Boyer and Filion (2007) show that oil price increases lead to higher stock returns of Canadian oil and gas companies. El-Sharif et al. (2005) reach the same conclusion when analyzing oil and gas returns in the UK. However, the authors note that non-oil and gas sectors are weakly linked to oil price changes. More recently, Nandha and Faff (2008) study the short-term link between oil prices and thirty-five Datastream global industries and report that oil price rises have a negative impact on all, but not the oil and gas industries. Finally, Nandha and Brooks (2009) look into the reaction of the transport sector to oil prices in thirty-eight countries and find that, in developed economies, oil prices have some influence on the returns of the sector under consideration. There is however no evidence of a significant role for oil price changes in Asian and Latin American countries. Taken together, the results from the available works on the relationships between oil price changes and sector stock returns are inconclusive and differ from country to country.

The current article extends the understanding of the relationship between oil price changes and stock returns at the disaggregated sector level in Europe by investigating their short-term linkages over the last turbulent decade using different econometric techniques. Over this decade of globally increasing oil prices, the responses of stock markets to oil price changes are ambiguous. Indeed, on the one hand increases in oil prices translate into higher transportation, production, and heating costs, which can put a drag on corporate earnings. Rising oil prices can also stir up concerns about inflation and curtail consumers' discretionary spending. On the other hand, investors can also associate increasing oil prices with a booming economy. Thus, higher oil prices could reflect stronger business performance.

It is equally important to note that studying the short-term effects of oil price fluctuations at sector level instead of aggregate market level is important for several reasons. First, any market-wide consequence may hide the performance, not necessarily uniform, of various economic sectors. Further, sector sensitivities to changes in oil price can be asymmetric to the extent that some sectors may be more severely affected by these changes than the others. The degree to which a sector is more or less sensitive to oil depend upon whether oil serves as its input or output, its exposure to the indirect oil effects, its degree of competition and concentration, and its capacity to absorb and transfer oil price risk to its consumers. Second, the industrial base varies from one European market to another. Large and mature markets 
such as France and Germany are more diversified, whereas small markets such as Switzerland usually concentrate on a few industries. Thus, the results of studies based on national stock market indices such as Park and Ratti (2008) and Apergis and Miller (2009) should be considered with precaution. An important and interesting issue consists then of examining how different sector market indices rather than national market indices react to oil price fluctuations. Finally, indentifying the heterogeneity of sector sensitivities to oil has important implications for portfolio risk management since some sectors may still provide a meaningful channel for international diversification during large swings in oil prices.

The rest of the paper is organized as follows. Section 2 presents the data and some preliminary analysis. Section 3 reports and discusses the empirical results. Section 4 focuses on some out-of-sample forecasting evaluations and portfolio implications of empirical results. Summary conclusions are provided in Section 5.

\section{Data and preliminary analysis}

We investigate the relationships between oil prices and stock returns in Europe from a sector perspective. Our sample data include the Dow Jones (DJ) Stoxx 600 and twelve European sector indices, namely Automobile \& Parts, Financials, Food \& Beverages, Oil \& Gas, Health Care, Industrials, Basic Materials, Personal \& Household Goods, Consumer Services, Technology, Telecommunications and Utilities. We collect stock market data from Datastream database.

Introduced in 1998, the Dow Jones Stoxx 600 sector indices aim to represent the largest European companies in each of the most important industries and currently cover Austria, Belgium, Denmark, Finland, France, Germany, Greece, Iceland, Ireland, Italy, Luxembourg, the Netherlands, Norway, Portugal, Spain, Sweden, Switzerland and the United Kingdom. The sector indices offer an alternative view of the performance of the European stock markets. The main industries are Automobile and Parts (automobiles, auto parts and tires), Financials (banks, insurance, reinsurance, real estate and financial services), Food and Beverages (beverages and food producers), Oil and Gas (oil and gas producers, oil equipment, and services, distribution and alternative energy), Health Care (health care equipment and services, and pharmaceuticals and biotechnology), Industrials (construction and materials, and industrial goods and services), Basic Materials (chemicals and basic resources), Personal and Household Goods (household goods, home construction, leisure goods, and personal goods and tobacco), Consumer Services (retail, media, travel and leisure), Technology (software and computer services, and technology hardware and equipment), Telecommunications (fixed line 
and mobile telecommunications), and Utilities (electricity, gas, water and multi-utilities). Each sector index represents a capitalization-weighted portfolio of the largest European companies in this sector.

We think that weekly data may better capture the interaction of oil and stock price changes than daily or monthly data. On the one hand, the use of weekly data in the analysis instead of daily data significantly reduces any potential biases that may arise such as the bid-ask effect, non-synchronous trading days, etc. On the other hand, the monthly data may have some bearing on asymmetry in responses of stock returns to oil price shocks. In this schema of thing, we make use of weekly stock market sector indices over the period from January 01, 1998 to November 13, 2008 and examine their sensitivity to the recent oil price boom after the 1997 Asian financial crisis. ${ }^{1}$ Over this sample period, the relationship between oil prices and stock markets was ambiguous as shown by Figure 1. Increases in oil prices were, at the same time, indicative of higher production costs and inflation pressure, and synonyms of higher expected economic growth and higher levels of consumer and investor confidence. Notice that weekly data running from November 20, 2008 to December 31, 2009 will be employed in our out-of-sample analysis to shed light on forecasting evaluation and some portfolio investment implications of the in-sample results.

For oil, we use the weekly Brent crude oil price obtained from the Energy Information Administration (EIA). The Europe Brent is one of the major international oil benchmarks. We express Brent oil prices in euro using euro/dollar exchange rates from Datastream.

Figure 1. European market index (DJ Stoxx 600) and crude oil price (Brent)

\footnotetext{
${ }^{1}$ It should be noted that both daily and monthly data as well as longer sample period are employed to subsequently check the robustness of our results.
} 


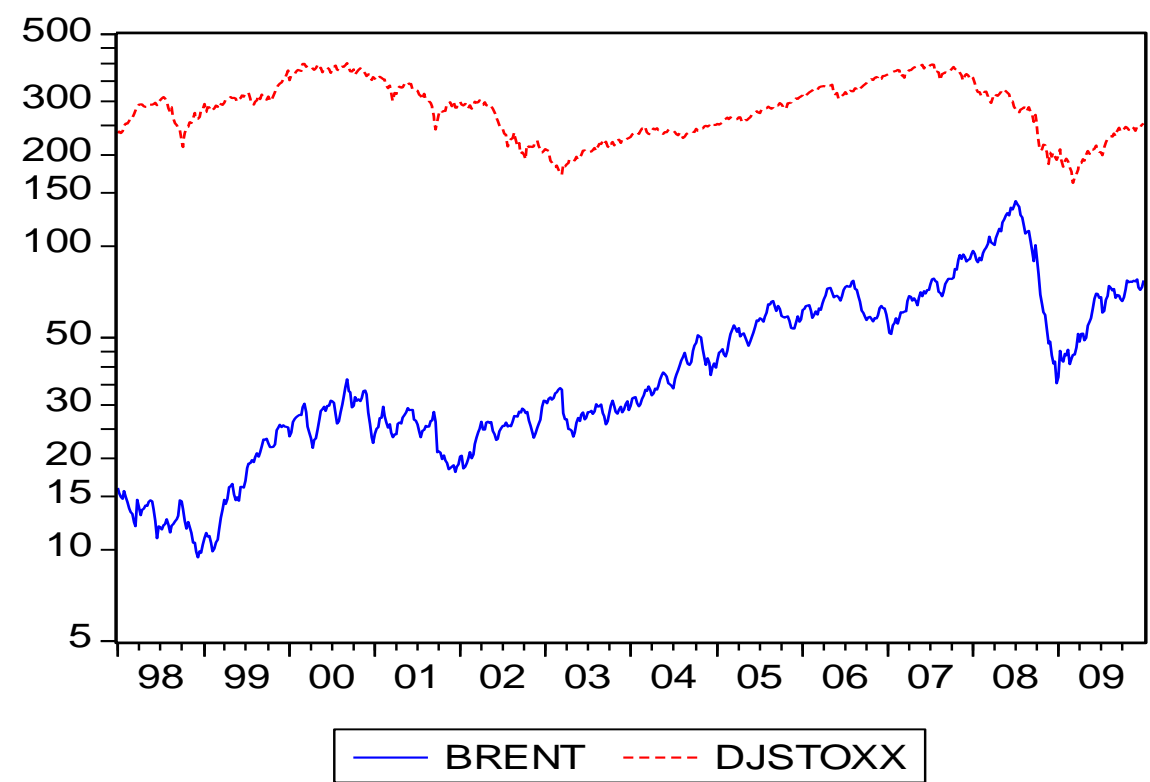

Before we can conduct further analysis on oil and stock market sector indices, the order of integration of our series is investigated using three standard unit root tests: Augmented Dickey-Fuller (ADF), Phillips-Perron (PP), and Kwiatkowski et al. (KPSS) tests. The ADF and PP tests are based on the null hypothesis of a unit root, while the KPSS test considers the null of no unit root. The obtained results are reported in Table 1. All the price series appear to be integrated of order one, which is a standard result in the literature for such series.

Table 1. Unit root tests

\begin{tabular}{|c|c|c|c|c|c|c|}
\hline & \multicolumn{3}{|c|}{ Levels } & \multicolumn{3}{|c|}{ First difference } \\
\hline & $\mathrm{ADF}$ & PP & KPSS & $\mathrm{ADF}$ & PP & KPSS \\
\hline Oil Brent & $0.60^{\mathrm{a}}$ & $-2.68^{b}$ & $0.69^{* * \mathrm{~b}}$ & $-11.48^{* a}$ & $-18.35^{* a}$ & $0.08^{b}$ \\
\hline DJ Stoxx & $-0.38^{\mathrm{a}}$ & $-0.26^{\mathrm{a}}$ & $0.37^{* \mathrm{~b}}$ & $-9.16^{* a}$ & $-23.6^{* a}$ & $0.27^{\mathrm{b}}$ \\
\hline Automobile \& Parts & $0.03^{\mathrm{a}}$ & $-2.14^{\mathrm{b}}$ & $0.67^{* * \mathrm{~b}}$ & $-27.48^{* a}$ & $-27.46^{* a}$ & $0.058^{\mathrm{b}}$ \\
\hline Financials & $-0.78^{a}$ & $-0.66^{\mathrm{c}}$ & $0.26^{\mathrm{b}}$ & $-8.29^{* a}$ & $-23.91^{*} \mathrm{c}$ & $0.35^{* \mathrm{~b}}$ \\
\hline Food \& Beverages & $0.43^{\mathrm{a}}$ & $-2.11^{\mathrm{b}}$ & $1.55^{* \mathrm{~b}}$ & $-21.83^{* a}$ & $21.83^{* a}$ & $0.08^{b}$ \\
\hline Oil \& Gas & $0.09^{\mathrm{a}}$ & $-2.01^{b}$ & $1.14^{* b}$ & $-15.54^{* a}$ & $-23.38^{* a}$ & $0.14^{\mathrm{b}}$ \\
\hline Health Care & $0.02^{\mathrm{a}}$ & $-2.07^{b}$ & $0.22^{\mathrm{b}}$ & $-25.85^{* a}$ & $-25.87^{* a}$ & $0.12^{\mathrm{b}}$ \\
\hline Industrials & $-0.48^{\mathrm{a}}$ & $-0.25^{\mathrm{a}}$ & $0.49^{* * \mathrm{~b}}$ & $-5.83^{* a}$ & $-22.12^{* a}$ & $0.19^{\mathrm{b}}$ \\
\hline Basic Materials & $0.34^{\mathrm{a}}$ & $0.32^{\mathrm{a}}$ & $2.03^{* b}$ & $-22.29^{* a}$ & $-22.47^{* a}$ & $0.14^{\mathrm{b}}$ \\
\hline Personal \& Household Goods & $0.30^{\mathrm{a}}$ & $1.87^{\mathrm{a}}$ & $1.55^{* \mathrm{~b}}$ & $-23.19^{* a}$ & $-23.23^{* a}$ & $0.18^{\mathrm{b}}$ \\
\hline Consumer Services & $-0.91^{\mathrm{a}}$ & $1.34^{\mathrm{c}}$ & $1.00^{* \mathrm{~b}}$ & $-6.21^{* a}$ & $-22.76^{* a}$ & $0.23^{\mathrm{b}}$ \\
\hline Technology & $-0.66^{\mathrm{a}}$ & $2.00^{\mathrm{b}}$ & $1.25^{* b}$ & $-6.49^{* a}$ & $-24.07^{* a}$ & $0.21^{\mathrm{b}}$ \\
\hline Telecommunications & $-0.30^{\mathrm{a}}$ & $-2.10^{\mathrm{c}}$ & $1.09^{* b}$ & $-7.79^{* a}$ & $-23.89^{* a}$ & $0.22^{b}$ \\
\hline Utilities & $0.75^{\mathrm{a}}$ & $0.76^{\mathrm{a}}$ & $1.45^{* \mathrm{~b}}$ & $17.4^{* \mathrm{a}}$ & $22.78^{*_{\mathrm{aa}}}$ & $0.19^{\mathrm{b}}$ \\
\hline
\end{tabular}

Notes: All variables are in natural logs. ADF is the Augmented Dickey-Fuller test, PP the Phillips-Perron test, and KPSS the Kwiatkowski-Phillips-Schmidt-Shin test. $\left({ }^{a}\right)$ indicates a model without constant or deterministic trend, $\left({ }^{b}\right)$ a model with constant without deterministic trend, and $\left(^{c}\right)$ a model with constant and deterministic trend. ${ }^{*},{ }^{* *}$ and ${ }^{* * *}$ denote rejection of the null hypothesis at the $1 \%, 5 \%$ and $10 \%$ levels respectively.

Descriptive statistics for return series (first logarithmic differences) are summarized in Table 2. On average, oil experienced higher returns than European stock market returns over our sample period. Technology stocks have the highest volatility followed by oil and Automobile stocks. Skewness is negative in most cases and the Jarque-Bera test statistic (JB) 
strongly rejects the hypothesis of normality. There is also strong evidence of ARCH effects and there are significant serial correlations for some series.

Table 2. Descriptive statistics of return series

\begin{tabular}{|c|c|c|c|c|c|c|c|c|c|c|}
\hline & Mean & $\begin{array}{l}\text { Std. } \\
\text { dev. }\end{array}$ & Skew. & Kurt. & $\begin{array}{l}\mathrm{ARCH} \\
\text { test }\end{array}$ & LB & $\mathrm{LB}^{2}$ & $\mathrm{JB}$ & $\begin{array}{l}\text { Corr. with } \\
\text { oil }\end{array}$ & $\begin{array}{l}\text { Corr. with } \\
\text { DJ Stoxx }\end{array}$ \\
\hline Oil Brent & 0.21 & 4.61 & -0.47 & 4.72 & $23.81^{*}$ & $38.07^{*}$ & $29.63^{*}$ & $91.4^{*}$ & 1.00 & 0.10 \\
\hline DJ Stoxx & -0.02 & 2.56 & -0.68 & 6.21 & $79.08^{*}$ & $10.62^{* * *}$ & $116.36^{*}$ & $291.9^{*}$ & 0.10 & 1.00 \\
\hline $\begin{array}{l}\text { Automobile and } \\
\text { Parts }\end{array}$ & 0.02 & 4.32 & 0.54 & 18.80 & $127.28^{*}$ & $11.81^{* * *}$ & $143.77^{*}$ & $5930.1^{*}$ & -0.02 & 0.67 \\
\hline Financials & -0.09 & 3.29 & -0.53 & 7.53 & $118.60^{*}$ & 8.99 & $231.09^{*}$ & $512.7^{*}$ & 0.04 & 0.92 \\
\hline Food and Beverages & 0.05 & 2.18 & -0.54 & 5.82 & $27.27^{*}$ & 7.45 & $36.10^{*}$ & $217.0^{*}$ & -0.10 & 0.57 \\
\hline Oil and Gas & 0.01 & 3.18 & -0.52 & 4.66 & $101.88^{*}$ & $15.16^{* *}$ & $187.45^{*}$ & $91.3^{*}$ & 0.33 & 0.63 \\
\hline Health Care & 0.01 & 2.54 & -0.03 & 5.23 & $44.93^{*}$ & 9.72 & $61.04^{*}$ & $118.5^{*}$ & -0.07 & 0.63 \\
\hline Industrials & -0.02 & 2.90 & -0.71 & 6.52 & $81.71^{*}$ & $12.68^{* *}$ & $98.56^{*}$ & $341.6^{*}$ & 0.10 & 0.91 \\
\hline Basic Materials & 0.06 & 3.34 & -0.90 & 7.02 & $140.87^{*}$ & 7.92 & $230.38^{*}$ & $459.5^{*}$ & 0.12 & 0.76 \\
\hline $\begin{array}{l}\text { Personal and } \\
\text { Household Goods }\end{array}$ & 0.04 & 2.57 & -0.76 & 6.70 & $40.53^{*}$ & 6.94 & $50.67^{*}$ & $378.6^{*}$ & 0.03 & 0.97 \\
\hline Consumer Services & -0.09 & 2.65 & -0.48 & 5.25 & $35.63^{*}$ & 7.68 & $49.48^{*}$ & $142.9^{*}$ & 0.04 & 0.88 \\
\hline Technology & -0.11 & 5.62 & -0.24 & 4.70 & $84.55^{*}$ & $13.52^{* *}$ & $118.03^{*}$ & $73.8^{*}$ & 0.08 & 0.81 \\
\hline Telecommunications & -0.01 & 3.63 & -0.07 & 4.01 & $72.18^{*}$ & $15.03^{* *}$ & $125.98^{*}$ & $24.7^{*}$ & 0.04 & 0.73 \\
\hline Utilities & 0.07 & 2.25 & -1.57 & 13.08 & $26.84^{*}$ & 4.09 & $36.58^{*}$ & $2635.3^{*}$ & 0.08 & 0.72 \\
\hline
\end{tabular}

Notes: this table reports the basic statistics of return series, including mean (Mean), standard deviations (Std. dev.) skewness (Skew.), and kurtosis (Kurt.). ARCH test is the statistical test for conditional heteroscedasticity of order 6. LB and $\mathrm{LB}^{2}$ are the Ljung-Box tests for autocorrelations of order 6 for the returns and for the squared returns. JB is the Jarque-Bera test for normality based on skewness and excess kurtosis. Corr. denotes the correlation coefficients. ${ }^{*},{ }^{* *}$ and ${ }^{* * *}$ indicate the rejection of the null hypothesis of associated statistical tests at the $1 \%, 5 \%$ and $10 \%$ levels respectively.

Correlations between oil price changes and European sector returns are generally weak, and surprisingly they are all positive, except for the three following sectors: Automobile and Parts, Food and Beverages, and Health Care. This suggests that oil price increases over the last decade were likely to be seen as an indicator of higher expected economic growth and earnings. The sector Oil and Gas has the highest degree of comovement with oil (0.33), followed by the sector Basic Materials (0.12). Correlations between the European market index (DJ Stoxx 600) and sector returns are high on average. The Personal and Household Goods sector shows the highest correlation (0.97) and the sector Food \& Beverages the lowest one (0.57).

\section{Empirical analysis}


We investigate the relationships between oil price changes and sector stock market returns in Europe over the last turbulent decade. We begin our analysis with the estimation of multifactor asset pricing models to investigate the sensitivities of the sector stock returns to oil price and European market changes, then we perform the Granger causality tests to examine their causal linkages, and finally we study cyclical comovements.

\subsection{Sector returns, oil price changes and market sensitivities}

In this subsection, the analysis is conducted as follows. First, we estimate a conditional version of the European market model for each sector. Second, we examine a two-factor model by introducing oil unexpected returns into the market model. The objective is to investigate sector return sensitivities to oil price shocks. Finally, we test for asymmetric interactions between oil price changes and European sector returns.

\section{a) The market model}

A conditional version of the European market model can be written as follows (Model 1): ${ }^{2}$

$$
\begin{aligned}
& r_{i t}=a+c \times r d j_{t}+\varepsilon_{i t} \\
& \varepsilon_{i t} \rightarrow f\left(0, h_{i t}\right) \\
& h_{i t}^{2}=\alpha+\beta_{k} \times \sum_{k=1}^{q} \varepsilon_{i, t-1}^{2}+\gamma_{l} \times \sum_{l=1}^{p} h_{i, t-1}^{2}
\end{aligned}
$$

where

$r_{i t}$ is the weekly stock returns for sector $i$;

$r d j_{t}$ represents the European stock market returns;

$\varepsilon_{i t}$ refers to a stochastic error term which is assumed to follow a $\operatorname{GARCH}(q, p)$ dynamics. $p$ and $q$ are explicitly determined according to commonly used information criteria.

$f($.$) is the density function of \varepsilon_{i t}$.

Model 1 is estimated for each of the considered sectors using the quasi-maximum likelihood (QML) method based on the Gaussian distribution. Here we also employ the Student's t-distribution to capture the distribution of sector returns because most series are highly skewed and exhibit significant excess kurtosis, leading to the rejection of normality. ${ }^{3}$

\footnotetext{
${ }^{2}$ When estimating our market models, an AR(1) term is used wherever it appears to be significant.

${ }^{3}$ Note however that the use of the Student's t-distribution is motivated by comparative purpose and thus does not disprove the results from assuming the normal distribution since the QML estimator is consistent and asymptotically normal under certain regularity conditions, even if the normality assumption is violated (Bollerslev and Wooldridge, 1992).
} 
We summarize the results in Table 3. As we can see, the estimates of model's parameters are somewhat similar, whatever the return distribution was used. However, according to the AIC information criteria, the model estimated using the Student's t-distribution shows superior results in 10 out of 12 cases. The evidence is mixed according to R-squared and LB criteria. The coefficients relating the sector returns to the European stock market returns (coefficients $c$ ) are highly significant for all sectors. They vary from 0.50 (defensive sector) for Food and Beverages to 1.46 (offensive sector) for Technology. The R-squared coefficients range from 31\% (Food and Beverages) to 82\% (Financials). The models we estimated seem to satisfactorily fit the data. The ARCH and GARCH coefficients are significant. We further observe that in most cases, conditional volatility does not change very sharply as the ARCH coefficients are relatively small in size. By contrast, it tends to fluctuate gradually over time because of the large GARCH coefficients. Note also that the estimates coefficients $\gamma$ and $\beta$ satisfy the stationary conditions. 
Table 3. Estimation results of the European market model

\begin{tabular}{|c|c|c|c|c|c|c|c|c|c|c|c|c|}
\hline Sectors & Distribution & $a$ & $c$ & $\alpha$ & $\beta_{1}$ & $\gamma_{1}$ & $\bar{R}^{2}$ & AIC & $\begin{array}{l}\mathrm{ARCH} \\
\text { test }\end{array}$ & $L B$ & $L B^{2}$ & $J B$ \\
\hline \multirow[t]{2}{*}{$\begin{array}{l}\text { Automobiles and } \\
\text { Parts }\end{array}$} & Normal & $\begin{array}{c}0.001 \\
(0.001)\end{array}$ & $\begin{array}{l}1.232^{*} \\
(0.041)\end{array}$ & $\begin{array}{l}0.001^{*} \\
(0.000)\end{array}$ & $\begin{array}{l}0.193^{*} \\
(0.057)\end{array}$ & $\begin{array}{l}0.752^{*} \\
(0.061)\end{array}$ & 0.455 & -4.558 & 4.900 & 6.219 & 5.314 & $83.115^{+}$ \\
\hline & Student- $t$ & $\begin{array}{c}0.000 \\
(0.000)\end{array}$ & $\begin{array}{l}1.238^{*} \\
(0.036)\end{array}$ & $\begin{array}{l}0.001^{* * *} \\
(0.000)\end{array}$ & $\begin{array}{l}0.184^{*} \\
(0.051)\end{array}$ & $\begin{array}{l}0.755^{*} \\
(0.068)\end{array}$ & 0.455 & -4.636 & 5.857 & 6.461 & 6.459 & $84.942^{+}$ \\
\hline \multirow[t]{2}{*}{ Financials } & Normal & $\begin{array}{c}-0.001^{* * * *} \\
(0.001)\end{array}$ & $\begin{array}{l}1.189^{*} \\
(0.025)\end{array}$ & $\begin{array}{l}0.001^{*} \\
(0.000)\end{array}$ & $\begin{array}{l}0.542^{*} \\
(0.120)\end{array}$ & - & 0.823 & -6.184 & 2.357 & 7.154 & 2.322 & $26.839^{+}$ \\
\hline & Student- $t$ & $\begin{array}{l}-0.001^{* *} \\
(0.000)\end{array}$ & $\begin{array}{l}1.178^{*} \\
(0.013)\end{array}$ & $\begin{array}{l}0.001^{*} \\
(0.000)\end{array}$ & $\begin{array}{l}0.625^{*} \\
(0.223)\end{array}$ & - & 0.822 & -6.205 & 2.211 & 6.766 & 2.198 & $27.020^{+}$ \\
\hline \multirow[t]{2}{*}{$\begin{array}{l}\text { Food and } \\
\text { Beverages }\end{array}$} & Normal & $\begin{array}{c}0.001 \\
(0.001)\end{array}$ & $\begin{array}{l}0.504^{*} \\
(0.038)\end{array}$ & $\begin{array}{l}0.000^{*} \\
(0.000)\end{array}$ & $\begin{array}{l}0.214^{*} \\
(0.073)\end{array}$ & - & 0.313 & -5.505 & 8.289 & 2.247 & 8.315 & $26.375^{+}$ \\
\hline & Student- $t$ & $\begin{array}{l}0.001^{* * * *} \\
(0.001) \\
\end{array}$ & $\begin{array}{l}0.511^{*} \\
(0.021) \\
\end{array}$ & $\begin{array}{c}0.000^{*} \\
(0.000) \\
\end{array}$ & $\begin{array}{l}0.295^{*} \\
(0.115) \\
\end{array}$ & - & 0.311 & -5.517 & 8.859 & 2.091 & 8.894 & $31.315^{+}$ \\
\hline \multirow[t]{2}{*}{ Oil and Gas } & Normal & $\begin{array}{c}0.000 \\
(0.001)\end{array}$ & $\begin{array}{l}0.793^{*} \\
(0.047)\end{array}$ & $\begin{array}{l}0.001^{*} \\
(0.000)\end{array}$ & $\begin{array}{l}0.202^{*} \\
(0.090)\end{array}$ & - & 0.410 & -4.652 & 3.626 & 6.732 & 3.552 & $12.877^{+}$ \\
\hline & Student- $t$ & $\begin{array}{c}0.000 \\
(0.001)\end{array}$ & $\begin{array}{c}0.799^{*} \\
(0.034)\end{array}$ & $\begin{array}{l}0.001^{*} \\
(0.000)\end{array}$ & $\begin{array}{l}0.206^{*} \\
(0.077)\end{array}$ & - & 0.410 & -4.613 & 3.374 & 6.823 & 3.302 & $15.309^{+}$ \\
\hline \multirow[t]{2}{*}{ Health Care } & Normal & $\begin{array}{c}0.000 \\
(0.001)\end{array}$ & $\begin{array}{l}0.596^{*} \\
(0.038)\end{array}$ & $\begin{array}{l}0.001^{*} \\
(0.000)\end{array}$ & $\begin{array}{l}0.066^{*} \\
(0.024)\end{array}$ & $\begin{array}{l}0.927^{*} \\
(0.027)\end{array}$ & 0.363 & -5.131 & 2.798 & 4.380 & 2.786 & $185.465^{+}$ \\
\hline & Student- $t$ & $\begin{array}{c}0.001 \\
(0.001) \\
\end{array}$ & $\begin{array}{l}0.641^{*} \\
(0.026) \\
\end{array}$ & $\begin{array}{c}0.001^{*} \\
(0.000)\end{array}$ & $\begin{array}{l}0.061^{*} \\
(0.026)\end{array}$ & $\begin{array}{l}0.938^{*} \\
(0.019) \\
\end{array}$ & 0.361 & -5.201 & 3.066 & 4.826 & 3.030 & $264.367^{+}$ \\
\hline \multirow[t]{2}{*}{ Industrials } & Normal & $\begin{array}{l}0.000 \\
(0.001)\end{array}$ & $\begin{array}{l}1.097^{*} \\
(0.025)\end{array}$ & $\begin{array}{l}0.001^{*} \\
(0.000)\end{array}$ & $\begin{array}{l}0.092^{*} \\
(0.024)\end{array}$ & $\begin{array}{l}0.901^{*} \\
(0.022)\end{array}$ & 0.809 & -6.107 & 1.769 & 2.901 & 1.800 & $25.343^{+}$ \\
\hline & Student- $t$ & $\begin{array}{c}0.000 \\
(0.001)\end{array}$ & $\begin{array}{l}1.078^{*} \\
(0.017)\end{array}$ & $\begin{array}{l}0.001^{*} \\
(0.000)\end{array}$ & $\begin{array}{l}0.090^{*} \\
(0.026)\end{array}$ & $\begin{array}{l}0.903^{*} \\
(0.025)\end{array}$ & 0.802 & -6.083 & 2.177 & 2.775 & 2.221 & $30.836^{+}$ \\
\hline \multirow[t]{2}{*}{ Basic Materials } & Normal & $\begin{array}{l}0.002^{*} \\
(0.001)\end{array}$ & $\begin{array}{l}1.100^{*} \\
(0.050)\end{array}$ & $\begin{array}{l}0.000^{*} \\
(0.000)\end{array}$ & $\begin{array}{l}0.522^{*} \\
(0.122)\end{array}$ & - & 0.520 & -5.068 & 2.219 & 3.960 & 2.197 & $97.920^{+}$ \\
\hline & Student- $t$ & $\begin{array}{l}0.001^{* *} \\
(0.001) \\
\end{array}$ & $\begin{array}{l}1.042^{*} \\
(0.029) \\
\end{array}$ & $\begin{array}{c}0.000^{*} \\
(0.000) \\
\end{array}$ & $\begin{array}{l}0.501^{*} \\
(0.119) \\
\end{array}$ & - & 0.524 & -5.094 & 1.992 & 3.864 & 1.976 & $141.832^{+}$ \\
\hline \multirow[t]{2}{*}{$\begin{array}{l}\text { Personal and } \\
\text { Household Goods }\end{array}$} & Normal & $\begin{array}{l}0.001^{*} \\
(0.001)\end{array}$ & $\begin{array}{l}0.896^{*} \\
(0.023)\end{array}$ & $\begin{array}{l}0.001^{*} \\
(0.000)\end{array}$ & $\begin{array}{l}0.081^{*} \\
(0.043)\end{array}$ & $\begin{array}{l}0.893^{*} \\
(0.040)\end{array}$ & 0.750 & -6.094 & 3.160 & 6.119 & 3.145 & $19.249^{+}$ \\
\hline & Student- $t$ & $\begin{array}{l}0.001^{*} \\
(0.001)\end{array}$ & $\begin{array}{l}0.886^{*} \\
(0.016) \\
\end{array}$ & $\begin{array}{l}0.001^{*} \\
(0.000)\end{array}$ & $\begin{array}{l}0.080^{*} \\
(0.022) \\
\end{array}$ & $\begin{array}{l}0.901^{*} \\
(0.028)\end{array}$ & 0.751 & -6.105 & 2.928 & 6.033 & 3.683 & $22.386^{+}$ \\
\hline \multirow[t]{2}{*}{ Consumer Services } & Normal & $\begin{array}{c}0.000 \\
(0.001)\end{array}$ & $\begin{array}{l}0.925^{*} \\
(0.018)\end{array}$ & $\begin{array}{l}0.000^{*} \\
(0.000)\end{array}$ & $\begin{array}{l}0.083^{*} \\
(0.035)\end{array}$ & $\begin{array}{l}0.901^{*} \\
(0.036)\end{array}$ & 0.766 & -6.092 & 2.243 & 5.596 & 2.323 & $76.473^{+}$ \\
\hline & Student- $t$ & $\begin{array}{c}0.000 \\
(0.001)\end{array}$ & $\begin{array}{l}0.917^{*} \\
(0.019)\end{array}$ & $\begin{array}{c}0.000^{*} \\
(0.000)\end{array}$ & $\begin{array}{l}0.062^{*} \\
(0.019)\end{array}$ & $\begin{array}{c}0.920^{*} \\
(0.022) \\
\end{array}$ & 0.766 & -6.137 & 2.888 & 5.773 & 3.016 & $90.125^{+}$ \\
\hline Technology & Normal & $\begin{array}{c}-0.000 \\
(0.001)\end{array}$ & $\begin{array}{c}1.460^{*} \\
(0.058)\end{array}$ & $\begin{array}{c}0.001^{*} \\
(0.000)\end{array}$ & $\begin{array}{c}0.080^{*} \\
(0.023)\end{array}$ & $\begin{array}{c}0.901^{*} \\
(0.030)\end{array}$ & 0.661 & -4.403 & 1.198 & 4.432 & 1.230 & $110.020^{+}$ \\
\hline
\end{tabular}




\begin{tabular}{|c|c|c|c|c|c|c|c|c|c|c|c|c|}
\hline & Student- $t$ & $\begin{array}{l}-0.000 \\
(0.001) \\
\end{array}$ & $\begin{array}{l}1.395^{*} \\
(0.036) \\
\end{array}$ & $\begin{array}{c}0.001^{*} \\
(0.000) \\
\end{array}$ & $\begin{array}{c}0.080^{*} \\
(0.027) \\
\end{array}$ & $\begin{array}{l}0.900^{*} \\
(0.027) \\
\end{array}$ & 0.656 & -4.444 & 1.038 & 4.985 & 1.059 & $200.180^{+}$ \\
\hline \multirow[t]{2}{*}{$\begin{array}{l}\text { Telecommunica- } \\
\text { tions }\end{array}$} & Normal & $\begin{array}{c}0.001 \\
(0.001)\end{array}$ & $\begin{array}{l}0.973^{*} \\
(0.036)\end{array}$ & $\begin{array}{l}0.000^{*} \\
(0.000)\end{array}$ & $\begin{array}{l}0.070^{*} \\
(0.014)\end{array}$ & $\begin{array}{l}0.911^{*} \\
(0.013)\end{array}$ & 0.517 & -4.761 & 2.661 & $13.350^{++}$ & 2.665 & $72.107^{+}$ \\
\hline & Student- $t$ & $\begin{array}{l}-0.000 \\
(0.001) \\
\end{array}$ & $\begin{array}{l}0.975^{*} \\
(0.035) \\
\end{array}$ & $\begin{array}{l}0.000^{*} \\
(0.000)\end{array}$ & $\begin{array}{l}0.088^{*} \\
(0.025) \\
\end{array}$ & $\begin{array}{l}0.907^{*} \\
(0.021) \\
\end{array}$ & 0.517 & -4.801 & 3.192 & $12.645^{++}$ & 3.150 & $102.450^{+}$ \\
\hline \multirow[t]{2}{*}{ Utilities } & Normal & $\begin{array}{l}0.001^{* * *} \\
(0.001)\end{array}$ & $\begin{array}{l}0.603^{*} \\
(0.029)\end{array}$ & $\begin{array}{l}0.001^{* * *} \\
(0.001)\end{array}$ & $\begin{array}{l}0.165^{*} \\
(0.063)\end{array}$ & $\begin{array}{l}0.721^{*} \\
(0.079)\end{array}$ & 0.528 & -5.554 & 5.710 & 6.039 & 5.961 & $101.720^{+}$ \\
\hline & Student- $t$ & $\begin{array}{l}0.001^{* *} \\
(0.001)\end{array}$ & $\begin{array}{l}0.604^{*} \\
(0.023)\end{array}$ & $\begin{array}{l}0.001^{* *} \\
(0.001)\end{array}$ & $\begin{array}{l}0.114^{*} \\
(0.051)\end{array}$ & $\begin{array}{l}0.757^{*} \\
(0.111)\end{array}$ & 0.527 & -5.999 & 7.132 & 5.949 & 7.321 & $142.970^{+}$ \\
\hline
\end{tabular}

Notes: this table reports the results from estimating the European market model for sector returns. Numbers in parenthesis are robust standard errors. $L B$ and $L B^{2}$ are the Ljung-Box tests for autocorrelation of order 6 for the standardised residuals and for the squared residuals. ARCH test is the LM ARCH test for conditional heteroscedasticity of order 6 . JB is the Jarque-Bera test for normality. AIC is the Akaike Information Criterion. For Food and Beverages, Basic Materials, and Industrials sectors, the model is estimated with an AR(1) because the latter is significant. We also tested for GARCH effect in the mean equation, but the associated coefficients are not significant. The orders for the GARCH model are determined based on information criteria. The degree of freedom $v$ for the Student's t-distribution is significantly higher than 2 in all cases, suggesting that the distribution of the standardized errors departs significantly from normality.

, and indicate the significance of coefficients at the $1 \%, 5 \%$ and $10 \%$ levels respectively.

$+{ }^{++}$and ${ }^{+++}$indicate the rejection of the null hypothesis of statistical tests at the $1 \%, 5 \%$ and $10 \%$ levels respectively. 
The Jarque-Bera statistics in Table 3 are considerably lower than those for the return series (Table 2). For instance, the JB statistic decreases from 5930.1 (Automobile and Parts), 2635.3 (Utilities) and 913.1 (Oil and Gas) to 83.1, 101.7 and 12.9 respectively. However, the normality hypothesis is still rejected indicating that the unconditional distribution of the conditional GARCH process is not sufficiently fat-tailed to accommodate the excess kurtosis in the data. This result justifies the use of the QML estimation method and the Student's tdistribution.

Finally, we also test for ARCH effects as well as for the absence of autocorrelation in the standardised residuals and in the squared residuals. The results indicate no serial correlations and heteroscedastic effects in the residuals, thus leading us to conclude that the model specification we use is flexible enough to capture the dynamics of returns.

\section{b) The two-factor "market and oil" model"}

Let us now consider an augmented version of the previous European market model by introducing the unexpected change in oil prices into Equation (1). This specification permits to assess the sensitivities of sector returns to oil price shocks and has the following form (Model 2): ${ }^{5}$

$$
\begin{aligned}
& r_{i t}=a+b \times r o i l_{t}^{u}+c \times r d j_{t}+\varepsilon_{i t} \\
& \varepsilon_{i t} \rightarrow f\left(0, h_{i t}\right) \\
& h_{i t}^{2}=\alpha+\beta_{k} \times \sum_{k=1}^{q} \varepsilon_{i, t-1}^{2}+\gamma_{l} \times \sum_{l=1}^{p} h_{i, t-1}^{2}
\end{aligned}
$$

where roil $_{t}^{u}$ is the unexpected change in oil prices, measured as the difference between the observed oil price change and the expected value of oil price change using the following regression model roil ${ }_{i t}=\mu+\sum_{l=1}^{k} \lambda_{l} \operatorname{roil}_{i, t-l}+\zeta_{i t}$

Obviously, the definition of unexpected changes in oil prices we retain in this paper would mean that the impact of previous oil price changes on stock returns is implicitly included in Equation (2). In this regard, the estimation results can be seen as a sort of causality tests

\footnotetext{
${ }^{4}$ We have also tested other multifactor models in which the relationships between oil and stock prices are controlled for by using other potential risk factors of stock returns. These factors include the changes in shortterm interest rates, the changes in consumer price index, and the changes in industrial production. Since the obtained results are very similar to our basic two-factor model as described by Equation (2), they are not reported here for concision purpose, but entirely available under request.

5 The suitability of two-factor "market and oil" pricing models, similar to the one we use in this paper, was empirically investigated in several past papers (see, e.g., Faff and Brailsford, 2000 and Arouri and Fouquau, 2009.
} 
between oil and stock returns. Based on information criteria, we retain three lags $(k=3)$ in order to appropriately remove autocorrelations in oil returns.

Table 4. Estimation results of the two-factor "market and oil" model

\begin{tabular}{|c|c|c|c|c|c|c|c|c|c|c|}
\hline Sectors & Distribution & $a$ & $b$ & $c$ & $\bar{R}^{2}$ & AIC & $\begin{array}{l}\mathrm{ARCH} \\
\text { test }\end{array}$ & $L B$ & $L B^{2}$ & $J B$ \\
\hline \multirow[t]{2}{*}{$\begin{array}{l}\text { Automobile } \\
\text { and Parts }\end{array}$} & Normal & $\begin{array}{c}0.001 \\
(0.001)\end{array}$ & $\begin{array}{c}-0.031 \\
(0.029)\end{array}$ & $\begin{array}{l}1.232^{*} \\
(0.042)\end{array}$ & 0.459 & -4.648 & 5.948 & 6.027 & 6.482 & $80.219^{+}$ \\
\hline & Student- $t$ & $\begin{array}{c}0.000 \\
(0.001)\end{array}$ & $\begin{array}{c}-0.033^{* * *} \\
(0.020)\end{array}$ & $\begin{array}{l}1.238^{*} \\
(0.036)\end{array}$ & 0.458 & -4.496 & 7.031 & 6.254 & 7.702 & $82.615^{+}$ \\
\hline \multirow[t]{2}{*}{ Financials } & Normal & $\begin{array}{c}0.000 \\
(0.000)\end{array}$ & $\begin{array}{l}0.029^{* *} \\
(0.015)\end{array}$ & $\begin{array}{l}1.170^{*} \\
(0.017)\end{array}$ & 0.851 & -6.341 & 2.823 & 6.804 & 2.666 & $32.726^{+}$ \\
\hline & Student- $t$ & $\begin{array}{r}-0.000 \\
(0.000) \\
\end{array}$ & $\begin{array}{l}0.031^{* *} \\
(0.017) \\
\end{array}$ & $\begin{array}{l}1.165^{*} \\
(0.015) \\
\end{array}$ & 0.851 & -6.350 & 2.211 & 6.199 & 2.335 & $49.002^{+}$ \\
\hline \multirow[t]{2}{*}{$\begin{array}{l}\text { Food and } \\
\text { Beverages }\end{array}$} & Normal & $\begin{array}{c}0.001 \\
(0.001)\end{array}$ & $\begin{array}{l}-0.049^{*} \\
(0.014)\end{array}$ & $\begin{array}{l}0.553^{*} \\
(0.026)\end{array}$ & 0.336 & -5.523 & 8.289 & 1.538 & 7.912 & $19.522^{+}$ \\
\hline & Student- $t$ & $\begin{array}{c}0.001 \\
(0.001)\end{array}$ & $\begin{array}{l}-0.049^{*} \\
(0.012)\end{array}$ & $\begin{array}{l}0.556^{*} \\
(0.022)\end{array}$ & 0.337 & -5.534 & 8.859 & 1.334 & 6.978 & $22.859^{+}$ \\
\hline \multirow[t]{2}{*}{ Oil and Gas } & Normal & $\begin{array}{l}-0.000 \\
(0.001)\end{array}$ & $\begin{array}{l}0.206^{*} \\
(0.019)\end{array}$ & $\begin{array}{l}0.820^{*} \\
(0.035)\end{array}$ & 0.505 & -4.851 & 4.830 & 7.376 & 4.797 & $21.222^{+}$ \\
\hline & Student- $t$ & $\begin{array}{c}0.000 \\
(0.001) \\
\end{array}$ & $\begin{array}{l}0.204^{*} \\
(0.019) \\
\end{array}$ & $\begin{array}{c}0.818^{*} \\
(0.033) \\
\end{array}$ & 0.505 & -4.864 & 4.843 & 7.818 & 4.776 & $15.309^{+}$ \\
\hline \multirow[t]{2}{*}{ Health Care } & Normal & $\begin{array}{l}-0.001 \\
(0.001)\end{array}$ & $\begin{array}{l}-0.075^{*} \\
(0.015)\end{array}$ & $\begin{array}{l}0.637^{*} \\
(0.026)\end{array}$ & 0.404 & -5.178 & 2.469 & 8.265 & 2.459 & $151.376^{+}$ \\
\hline & Student- $t$ & $\begin{array}{c}0.000 \\
(0.000) \\
\end{array}$ & $\begin{array}{l}-0.083^{*} \\
(0.018) \\
\end{array}$ & $\begin{array}{c}0.594^{*} \\
(0.037) \\
\end{array}$ & 0.405 & -5.243 & 2.567 & 7.259 & 2.548 & $215.882^{+}$ \\
\hline \multirow[t]{2}{*}{ Industrials } & Normal & $\begin{array}{c}0.001 \\
(0.001)\end{array}$ & $\begin{array}{l}0.016^{* * *} \\
(0.009)\end{array}$ & $\begin{array}{l}1.097^{*} \\
(0.025)\end{array}$ & 0.831 & -6.115 & 1.899 & 2.788 & 1.800 & $25.687^{+}$ \\
\hline & Student- $t$ & $\begin{array}{c}0.000 \\
(0.001) \\
\end{array}$ & $\begin{array}{c}0.0015^{* *} \\
(0.008)\end{array}$ & $\begin{array}{c}1.079^{*} \\
(0.017) \\
\end{array}$ & 0.832 & -6.128 & 2.436 & 2.650 & 2.340 & $33.006^{+}$ \\
\hline \multirow[t]{2}{*}{$\begin{array}{l}\text { Basic Mate- } \\
\text { rials }\end{array}$} & Normal & $\begin{array}{l}0.002^{* *} \\
(0.001)\end{array}$ & $\begin{array}{l}0.032^{* * *} \\
(0.015)\end{array}$ & $\begin{array}{l}1.085^{*} \\
(0.038)\end{array}$ & 0.600 & -5.069 & 2.113 & 3.553 & 2.137 & $78.634^{+}$ \\
\hline & Student- $t$ & $\begin{array}{l}0.002^{* *} \\
(0.001)\end{array}$ & $\begin{array}{c}0.025^{* * *} \\
(0.014) \\
\end{array}$ & $\begin{array}{c}1.050^{*} \\
(0.028) \\
\end{array}$ & 0.601 & -5.095 & 1.891 & 3.252 & 1.893 & $115.556^{+}$ \\
\hline \multirow{2}{*}{$\begin{array}{l}\text { Personal and } \\
\text { Household } \\
\text { Goods }\end{array}$} & Normal & $\begin{array}{l}0.001^{* * *} \\
(0.001)\end{array}$ & $\begin{array}{l}-0.035 \\
(0.023)\end{array}$ & $\begin{array}{l}0.899^{*} \\
(0.023)\end{array}$ & 0.762 & -6.110 & 7.351 & 8.040 & 6.680 & $13.710^{+}$ \\
\hline & Student- $t$ & $\begin{array}{c}0.001^{* * *} \\
(0.001)\end{array}$ & $\begin{array}{l}-0.035 \\
(0.024) \\
\end{array}$ & $\begin{array}{c}0.891^{*} \\
(0.016) \\
\end{array}$ & 0.763 & -6.117 & 6.798 & 7.867 & 6.148 & $15.224^{+}$ \\
\hline \multirow[t]{2}{*}{$\begin{array}{l}\text { Consumer } \\
\text { Services }\end{array}$} & Normal & $\begin{array}{c}0.000 \\
(0.000)\end{array}$ & $\begin{array}{l}0.023^{* *} \\
(0.012)\end{array}$ & $\begin{array}{l}0.927^{*} \\
(0.018)\end{array}$ & 0.788 & -6.093 & 2.181 & 5.076 & 2.289 & $92.179^{+}$ \\
\hline & Student- $t$ & $\begin{array}{c}0.000 \\
(0.000) \\
\end{array}$ & $\begin{array}{l}0.025^{* *} \\
(0.013) \\
\end{array}$ & $\begin{array}{c}0.919^{*} \\
(0.018) \\
\end{array}$ & 0.788 & -6.143 & 3.110 & 5.367 & 3.276 & $107.801^{+}$ \\
\hline \multirow[t]{2}{*}{ Technology } & Normal & $\begin{array}{c}0.001 \\
(0.001)\end{array}$ & $\begin{array}{l}-0.043^{* *} \\
(0.022)\end{array}$ & $\begin{array}{l}1.452^{*} \\
(0.059)\end{array}$ & 0.690 & -4.486 & 1.023 & 4.189 & 1.032 & $120.890^{+}$ \\
\hline & Student- $t$ & $\begin{array}{r}-0.000 \\
(0.000) \\
\end{array}$ & $\begin{array}{c}-0.053^{* *} \\
(0.021) \\
\end{array}$ & $\begin{array}{l}1.387^{*} \\
(0.036) \\
\end{array}$ & 0.694 & -4.511 & 0.926 & 4.713 & 0.657 & $231.910^{+}$ \\
\hline \multirow[t]{2}{*}{$\begin{array}{l}\text { Telecommu- } \\
\text { nications }\end{array}$} & Normal & $\begin{array}{l}-0.001 \\
(0.001)\end{array}$ & $\begin{array}{l}-0.026 \\
(0.021)\end{array}$ & $\begin{array}{l}0.975^{*} \\
(0.032)\end{array}$ & 0.529 & -4.781 & 1.904 & $11.751^{+++}$ & 1.965 & $86.954^{+}$ \\
\hline & Student- $t$ & $\begin{array}{r}-0.000 \\
(0.001) \\
\end{array}$ & $\begin{array}{r}-0.018 \\
(0.022) \\
\end{array}$ & $\begin{array}{l}0.966^{*} \\
(0.034) \\
\end{array}$ & 0.528 & -4.810 & 2.416 & $9.806^{+++}$ & 2.423 & $149.860^{+}$ \\
\hline \multirow[t]{2}{*}{ Utilities } & Normal & $\begin{array}{c}0.001 \\
(0.001)\end{array}$ & $\begin{array}{c}0.008 \\
(0.013)\end{array}$ & $\begin{array}{l}0.603^{*} \\
(0.029)\end{array}$ & 0.529 & -5.558 & 5.718 & 5.246 & 6.086 & $98.310^{+}$ \\
\hline & Student- $t$ & $\begin{array}{l}0.001^{* * *} \\
(0.001)\end{array}$ & $\begin{array}{c}0.004 \\
(0.012) \\
\end{array}$ & $\begin{array}{l}0.605^{*} \\
(0.022) \\
\end{array}$ & 0.529 & -5.602 & 7.187 & 5.470 & 7.101 & $141.85^{+}$ \\
\hline
\end{tabular}

Notes: this table reports the results from estimating the tow-factor "market and oil" model for sector returns. Numbers in parenthesis are robust standard errors. $L B$ and $L B^{2}$ are the Ljung-Box tests for autocorrelation of order 6 for the standardised residuals and for the squared residuals. ARCH test is the LM ARCH test for conditional heteroscedasticity of order 6 . $J B$ is the Jarque-Bera test for normality. AIC is the Akaike Information Criterion. For Food and Beverages, Health Care, Basic Materials, and Industrials sectors, the model is estimated with an AR(1) because the latter is significant. We also tested for GARCH effect in the mean equation, but the associated coefficients are not significant. The orders for the GARCH model are determined based on information criteria. The degree of freedom $v$ for the Student's t-distribution is significantly higher than 2 in all cases, suggesting that the distribution of the standardized errors departs significantly from normality. The GARCH coefficients are not reported here in order to preserve space, but they are similar to those reported in Table 3. ${ }^{*},{ }^{* *}$ and ${ }^{* * *}$ indicate the significance of coefficients at the $1 \%, 5 \%$ and $10 \%$ levels respectively. 
${ }^{+},{ }^{++}$and ${ }^{+++}$indicate the rejection of the null hypothesis of statistical tests at the $1 \%, 5 \%$ and $10 \%$ levels respectively.

The estimation results are summarized in Table $4{ }^{6}$ The coefficients relating the return series to oil price changes (coefficients $b$ ) are significant in eight cases, indicating significant short-term effects of oil price fluctuations on European sector stock returns. Oil price increases negatively affect sector returns in three cases (Food and Beverages, Health Care and Technology), and positively in five cases (Financials, Oil and Gas, Industrials, Basic Materials, and Consumer Services), which is consistent with the correlations reported in Table 2. This confirms our intuition that, while higher oil prices imply lower stock returns for some industries due to higher production and transportation costs and lower corporate earnings, increases in oil prices over the last decade also reflect the increases in world demand for oil in response to periods of high economic growth, and thus lead to positive stock returns for other sectors. Additionally, the sign of the oil-stock price relationships is also likely to be dependent on the capacity of the industry to transfer oil price shocks to other economic entities, through for example hedging contracts on commodity derivatives markets, and thus to minimise the impact of these shocks on its profitability. Finally, our results show that there is no relationship between oil price changes and stock returns for three European sectors (Personal and Household Goods, Telecommunications, and Utilities), whereas for the Automobile and Parts industry a negative weak link is obtained.

It is equally important to note that whenever oil price changes are significant, the twofactor "market and oil" model outperforms the market model as the AIC and R-squared scores in Table 4 are respectively smaller and larger than those in Table 3.

Summarizing all, our analysis shows strong linkages between oil price changes and most European sector returns over the period under consideration. The sign and intensity of these linkages differ from one sector to another. In the following sub-section, we test for asymmetries in the responses of European sector returns to oil price shocks.

\section{c) Asymmetric reaction to oil shocks}

Some recent papers have shown that the link between oil and economic activity is not entirely linear and that negative oil price shocks (price increases) tend to have larger impacts on growth than positive shocks do (Hamilton, 2003; Lardic and Mignon, 2006; Zhang, 2008; Cologni and Manera, 2009). One should expect that oil price changes equally affect stock markets in an asymmetric fashion. To empirically test for asymmetry in the reaction of

\footnotetext{
${ }^{6}$ We do not report the estimates of the GARCH coefficients as they are very similar to those reported in Table 3 . Note also that when estimating the two-factor "market and oil" model, an autoregressive term AR(1) is used wherever it shows up as being significant.
} 
European sector returns to oil price shocks, we rely on the estimation of the following asymmetric multifactor model (Model 3):

$$
\begin{aligned}
& r_{i t}=a+b^{+} D_{t} \times \operatorname{roil}_{t}^{u}+b^{-}\left(1-D_{t}\right) \times \operatorname{roil}_{t}^{u}+c \times r d j_{t}+\varepsilon_{i t} \\
& \varepsilon_{i t} \rightarrow f\left(0, h_{i t}\right) \\
& h_{i t}^{2}=\alpha+\beta_{k} \times \sum_{k=1}^{q} \varepsilon_{i, t-1}^{2}+\gamma_{l} \times \sum_{l=1}^{p} h_{i, t-1}^{2}
\end{aligned}
$$

where $D_{t}$ is a dummy variable taking a value of one if unexpected change in oil price is positive and zero if it is negative. ${ }^{7}$ Accordingly, $b^{+}$and $b^{-}$are the coefficients corresponding to increases and decreases in unexpected oil price respectively. There is no asymmetry if $b^{+}$ and $b^{-}$are not statistically different from each other, which requires us to test the null hypothesis of coefficient equality, $b^{+}=b^{-}$. We are also interested in testing for the null hypothesis of non asymmetry and nonsensitivities to oil price increases and decreases, $b^{+}=b^{-}=0$.

Our main empirical results are summarized in Table 5. Wald tests show that the hypothesis $b^{+}=b^{-}=0$ is rejected mostly at the $1 \%$ level in nine cases, which confirms the significance of oil price shocks as a factor affecting sector returns in Europe. Oil price changes do not significantly affect stock returns in the Automobile and Parts, Telecommunications, and Utilities sectors. This is in line with our findings reported in Table 4. The only exception is for Personal and Household Goods for which no significant reaction to oil shocks is observed when the symmetric asset pricing model was used. Indeed, asymmetric results in Table 5 show that stock returns in this sector react negatively to unexpected oil price increases and negatively to expected oil price decreases. Wald tests confirm this finding and show that the hypothesis $b^{+}=b^{-}$is rejected for Personal and Household Goods as well as for two other sectors (Food and Beverages, and Health Care). This hypothesis is also weakly rejected at $10 \%$ for Basic Materials. For all these industries, reactions to oil price changes are asymmetric.

\footnotetext{
${ }^{7}$ Note that we have also estimated the asymmetric multifactor model with an $\operatorname{EGARCH}(1,1)$ process, but information criteria lead us to prefer the $\operatorname{GARCH}(q, p)$ specification we report in this paper.
} 
Table 5. Estimation results of the asymmetric asset pricing model

\begin{tabular}{|c|c|c|c|c|c|c|c|c|c|c|c|}
\hline Sectors & Distribution & $b^{+}$ & $b^{-}$ & $b^{+}=b^{-}=0$ & $b^{+}=b^{-}$ & $\bar{R}^{2}$ & AIC & $\begin{array}{c}\text { ARCH } \\
\text { Test }\end{array}$ & $L B$ & $L B^{2}$ & $J B$ \\
\hline \multirow[t]{2}{*}{$\begin{array}{l}\text { Automobile } \\
\text { and Parts }\end{array}$} & Normal & $\begin{array}{l}-0.026 \\
(0.040)\end{array}$ & $\begin{array}{c}-0.047 \\
(0.039)\end{array}$ & $\begin{array}{c}3.041 \\
{[0.218]}\end{array}$ & $\begin{array}{c}0.097 \\
{[0.755]}\end{array}$ & 0.460 & -4.645 & 6.100 & 6.040 & 6.686 & $79.930^{+}$ \\
\hline & Student- $t$ & $\begin{array}{l}-0.018 \\
(0.038) \\
\end{array}$ & $\begin{array}{r}-0.039 \\
(0.024) \\
\end{array}$ & $\begin{array}{c}2.026 \\
{[0.363]}\end{array}$ & $\begin{array}{c}0.099 \\
{[0.752]}\end{array}$ & 0.459 & -4.493 & 7.199 & 6.281 & 7.921 & $82.331^{+}$ \\
\hline \multirow[t]{2}{*}{ Financials } & Normal & $\begin{array}{c}0.016 \\
(0.018)\end{array}$ & $\begin{array}{l}0.040^{* *} \\
(0.016)\end{array}$ & $\begin{array}{l}11.475 \\
{[0.003]}\end{array}$ & $\begin{array}{c}0.646 \\
{[0.422]}\end{array}$ & 0.851 & -6.338 & 2.962 & 6.886 & 2.808 & $31.164^{+}$ \\
\hline & Student- $t$ & $\begin{array}{c}0.019 \\
(0.017) \\
\end{array}$ & $\begin{array}{l}0.042^{*} \\
(0.017)\end{array}$ & $\begin{array}{l}14.650 \\
{[0.001]}\end{array}$ & $\begin{array}{c}0.639 \\
{[0.423]}\end{array}$ & 0.851 & -6.357 & 2.211 & 6.302 & 2.474 & $46.543^{+}$ \\
\hline \multirow[t]{2}{*}{$\begin{array}{l}\text { Food and } \\
\text { Beverages }\end{array}$} & Normal & $\begin{array}{l}-0.071^{*} \\
(0.023)\end{array}$ & $\begin{array}{c}0.031 \\
(0.020)\end{array}$ & $\begin{array}{l}18.354 \\
{[0.000]}\end{array}$ & $\begin{array}{l}12.299 \\
{[0.000]}\end{array}$ & 0.343 & -5.532 & 7.109 & 1.522 & 6.545 & $16.032^{+}$ \\
\hline & Student- $t$ & $\begin{array}{c}-0.061^{* *} \\
(0.025) \\
\end{array}$ & $\begin{array}{c}0.039^{* * *} \\
(0.021)\end{array}$ & $\begin{array}{l}15.681 \\
{[0.000]}\end{array}$ & $\begin{array}{l}13.339 \\
{[0.000]}\end{array}$ & 0.343 & -5.541 & 6.768 & 1.336 & 6.355 & $22.859^{+}$ \\
\hline \multirow[t]{2}{*}{ Oil and Gas } & Normal & $\begin{array}{l}0.190^{*} \\
(0.042)\end{array}$ & $\begin{array}{l}0.221^{*} \\
(0.029)\end{array}$ & $\begin{array}{l}133.712 \\
{[0.000]}\end{array}$ & $\begin{array}{c}0.239 \\
{[0.624]}\end{array}$ & 0.505 & -4.828 & 4.419 & 7.122 & 4.660 & $21.300^{+}$ \\
\hline & Student- $t$ & $\begin{array}{l}0.190^{*} \\
(0.041)\end{array}$ & $\begin{array}{l}0.218^{*} \\
(0.030) \\
\end{array}$ & $\begin{array}{l}123.499 \\
{[0.000]}\end{array}$ & $\begin{array}{c}0.233 \\
{[0.629]}\end{array}$ & 0.505 & -4.841 & 4.732 & 7.590 & 4.661 & $25.141^{+}$ \\
\hline \multirow[t]{2}{*}{ Health Care } & Normal & $\begin{array}{l}-0.022 \\
(0.032)\end{array}$ & $\begin{array}{l}-0.134^{*} \\
(0.027)\end{array}$ & $\begin{array}{c}36.831 \\
{[0.000]}\end{array}$ & $\begin{array}{c}4.579 \\
{[0.032]}\end{array}$ & 0.457 & -5.192 & 2.216 & 6.172 & 2.874 & $150.063^{+}$ \\
\hline & Student- $t$ & $\begin{array}{c}-0.002 \\
(0.028)\end{array}$ & $\begin{array}{l}-0.135^{*} \\
(0.026)\end{array}$ & $\begin{array}{l}33.167 \\
{[0.000]}\end{array}$ & $\begin{array}{c}8.250 \\
{[0.004]}\end{array}$ & 0.457 & -5.291 & 2.785 & 6.292 & 2.750 & $206.987^{+}$ \\
\hline \multirow[t]{2}{*}{ Industrials } & Normal & $\begin{array}{c}-0.014 \\
(0.009)\end{array}$ & $\begin{array}{l}0.019^{* * *} \\
(0.012)\end{array}$ & $\begin{array}{l}11.130 \\
{[0.003]}\end{array}$ & $\begin{array}{c}0.974 \\
{[0.327]}\end{array}$ & 0.831 & -6.104 & 1.741 & 2.837 & 1.646 & $26.679^{+}$ \\
\hline & Student- $t$ & $\begin{array}{l}-0.023 \\
(0.016) \\
\end{array}$ & $\begin{array}{c}0.018^{* * *} \\
(0.011) \\
\end{array}$ & $\begin{array}{l}11.606 \\
{[0.002]}\end{array}$ & $\begin{array}{c}1.601 \\
{[0.206]}\end{array}$ & 0.833 & -6.128 & 2.355 & 2.703 & 2.244 & $34.203^{+}$ \\
\hline \multirow[t]{2}{*}{$\begin{array}{l}\text { Basic Mate- } \\
\text { rials }\end{array}$} & Normal & $\begin{array}{l}-0.015 \\
(0.032)\end{array}$ & $\begin{array}{l}0.073^{* *} \\
(0.029)\end{array}$ & $\begin{array}{c}16.282 \\
{[0.000]}\end{array}$ & $\begin{array}{c}2.806 \\
{[0.093]}\end{array}$ & 0.674 & -5.071 & 2.118 & 3.490 & 2.224 & $75.543^{+}$ \\
\hline & Student- $t$ & $\begin{array}{l}-0.017 \\
(0.029) \\
\end{array}$ & $\begin{array}{l}0.062^{* *} \\
(0.028) \\
\end{array}$ & $\begin{array}{l}19.058 \\
{[0.000]} \\
\end{array}$ & $\begin{array}{r}2.709 \\
{[0.099]} \\
\end{array}$ & 0.674 & -5.123 & 1.930 & 3.208 & 1.929 & $98.098^{+}$ \\
\hline \multirow{2}{*}{$\begin{array}{l}\text { Personal and } \\
\text { Household } \\
\text { Goods }\end{array}$} & Normal & $\begin{array}{l}-0.077^{*} \\
(0.021)\end{array}$ & $\begin{array}{l}0.051^{* *} \\
(0.026)\end{array}$ & $\begin{array}{l}16.580 \\
{[0.000]}\end{array}$ & $\begin{array}{c}5.026 \\
{[0.025]}\end{array}$ & 0.793 & -6.136 & 7.132 & 8.019 & 6.523 & $11.555^{+}$ \\
\hline & Student- $t$ & $\begin{array}{l}-0.074^{*} \\
(0.019) \\
\end{array}$ & $\begin{array}{l}0.051^{* *} \\
(0.025) \\
\end{array}$ & $\begin{array}{l}15.133 \\
{[0.000]} \\
\end{array}$ & $\begin{array}{r}6.030 \\
{[0.014]} \\
\end{array}$ & 0.793 & -6.222 & 7.011 & 7.768 & 6.099 & $13.054^{+}$ \\
\hline \multirow[t]{2}{*}{$\begin{array}{l}\text { Consumer } \\
\text { Services }\end{array}$} & Normal & $\begin{array}{c}0.032^{* * *} \\
(0.019)\end{array}$ & $\begin{array}{c}0.016 \\
(0.016)\end{array}$ & $\begin{array}{c}6.853 \\
{[0.032]}\end{array}$ & $\begin{array}{c}0.265 \\
{[0.696]}\end{array}$ & 0.788 & -6.090 & 2.174 & 5.083 & 2.284 & $90.565^{+}$ \\
\hline & Student- $t$ & $\begin{array}{c}0.031 \\
(0.021) \\
\end{array}$ & $\begin{array}{c}0.018 \\
(0.019) \\
\end{array}$ & $\begin{array}{c}5.968 \\
{[0.051]} \\
\end{array}$ & $\begin{array}{c}0.185 \\
{[0.666]}\end{array}$ & 0.788 & -6.140 & 2.907 & 5.123 & 2.934 & $103.886^{+}$ \\
\hline \multirow[t]{2}{*}{ Technology } & Normal & $\begin{array}{l}-0.010 \\
(0.032)\end{array}$ & $\begin{array}{l}-0.072^{* *} \\
(0.035)\end{array}$ & $\begin{array}{c}5.660 \\
{[0.059]}\end{array}$ & $\begin{array}{c}1.160 \\
{[0.281]}\end{array}$ & 0.691 & -4.487 & 1.184 & 4.121 & 1.200 & $113.765^{+}$ \\
\hline & Student- $t$ & $\begin{array}{l}-0.016 \\
(0.034) \\
\end{array}$ & $\begin{array}{l}-0.088^{*} \\
(0.031) \\
\end{array}$ & $\begin{array}{l}11.722 \\
{[0.003]}\end{array}$ & $\begin{array}{c}1.620 \\
{[0.203]}\end{array}$ & 0.694 & -4.518 & 0.909 & 4.332 & 0.765 & $208.123^{+}$ \\
\hline \multirow[t]{2}{*}{$\begin{array}{l}\text { Telecommu- } \\
\text { nications }\end{array}$} & Normal & $\begin{array}{c}0.031 \\
(0.042)\end{array}$ & $\begin{array}{c}-0.062 \\
(0.047)\end{array}$ & $\begin{array}{c}1.766 \\
{[0.413]}\end{array}$ & $\begin{array}{c}1.361 \\
{[0.243]}\end{array}$ & 0.530 & -4.386 & 1.768 & $11.618^{+++}$ & 1.858 & $81.098^{+}$ \\
\hline & Student- $t$ & $\begin{array}{l}-0.001 \\
(0.042) \\
\end{array}$ & $\begin{array}{c}-0.035 \\
(0.040) \\
\end{array}$ & $\begin{array}{c}0.947 \\
{[0.622]}\end{array}$ & $\begin{array}{c}0.244 \\
{[0.621]}\end{array}$ & 0.529 & -4.427 & 1.978 & $10.112^{+++}$ & 2.098 & $145.768^{+}$ \\
\hline \multirow[t]{2}{*}{ Utilities } & Normal & $\begin{array}{c}0.001 \\
(0.001)\end{array}$ & $\begin{array}{c}-0.008 \\
(0.013)\end{array}$ & $\begin{array}{c}0.678 \\
{[0.712]}\end{array}$ & $\begin{array}{c}0.495 \\
{[0.481]}\end{array}$ & 0.530 & -5.556 & 5.871 & 5.192 & 6.332 & $88.786^{+}$ \\
\hline & Student- $t$ & $\begin{array}{l}-0.005 \\
(0.024) \\
\end{array}$ & $\begin{array}{l}-0.0041 \\
(0.022) \\
\end{array}$ & $\begin{array}{c}0.122 \\
{[0.940]} \\
\end{array}$ & $\begin{array}{c}0.001 \\
{[0.977]}\end{array}$ & 0.529 & -5.599 & 7.875 & 5.445 & 6.654 & $132.988^{+}$ \\
\hline
\end{tabular}

Notes: this table reports the results from estimating the asset pricing model with asymmetric reaction of sector returns to oil price shocks. Numbers in parenthesis are robust standard errors. In columns 5 and 6, we report empirical statistics of the Wald tests and their associated $p$-values in brackets. $L B$ and $L B^{2}$ are the Ljung-Box tests for autocorrelation of order 6 for the standardised residuals and for the squared residuals. ARCH test is the LM ARCH test for conditional heteroscedasticity of order 6 . $J B$ is the Jarque-Bera test for normality. AIC is the Akaike Information Criterion. For Food and Beverages, Health Care, Basic Materials, and Industrials sectors, the model is estimated with an AR(1) because the latter is significant. We also tested for GARCH effect in the mean equation, but the associated coefficients are not significant. The orders for the GARCH model are determined based on information criteria. The degree of freedom $v$ for the Student's t-distribution is significantly higher than 2 in all cases, suggesting that the distribution of the standardized errors departs significantly from normality. The GARCH coefficients are not reported for concision purpose, but they are similar to those reported in Table $3 .{ }^{*},{ }^{* *}$ and ${ }^{* * *}$ indicate the significance of coefficients at the $1 \%, 5 \%$ and $10 \%$ levels respectively. ${ }^{+},{ }^{++}$and ${ }^{++}$indicate the rejection of the null hypothesis of statistical tests at the $1 \%, 5 \%$ and $10 \%$ levels respectively. 
Finally, it is worth noting that according the different criteria used to chose the most appropriate model (R-squared, AIC, LB, etc.), the asymmetric asset pricing model appears to be the best one when there is presence of asymmetry in the relationship between oil and stock returns, i.e., for these four industries Personal and Household Goods, Food and Beverages, Basic Materials, and Health Care. Also, the models incorporating oil returns (i.e., Models 2 3) shows superior results to the market model (i.e., Model 1) for all the industries for which oil price changes are priced, the only exceptions being Telecommunications, and Utilities sectors. All in all, these findings suggest that oil price changes play a significant role in explaining stock returns in most European industries and that there are evidence to show that some sector returns respond asymmetrically to the impact of oil price changes.

\subsection{Causality tests}

In order to further examine the relationships between oil price changes and sector stock returns in Europe, we proceed with testing for Granger causality between return series. Results are reported in Table 6. Since some variables as well as their bilateral effects are very sensitive to the selected number of lags in the analysis, this test is implemented for different lags.

The results show that there is bidirectional causality between oil price changes and DJ Stoxx returns. Indeed, DJ Stoxx returns granger-cause changes in oil prices at the $10 \%$ level for from one to three autoregressive lags, whereas oil price shocks granger-cause changes in DJ Stoxx returns at the 5\% level for all lags, except the first one. Similar results are obtained for different lags with regard to Automobile and Parts, Food and Beverages, Oil and Gas, Industrials, Personal and Household Goods, Consumer Services and Utilities industries. Unidirectional Granger causality from oil to stock returns is significant for Financials (at the 10\% level for lags 2 and 8), Health Care (at the 10\% level only for lag 2), and Technology (at the $10 \%$ level for lag 2 and at the 5\% for lags 8,10 and 12), while causality from stock returns to oil is only found to be significant for Basic Materials (at the 1\% for all lags). Finally, there is absence of significant causality between oil price changes and stock returns in Telecommunications sector.

Taken together, the results of our causality tests corroborate our previous findings and suggest significant interactions between oil prices and stock prices, except for Telecommunications stocks. These results are interesting at least for two reasons. First, they imply some predictability in oil and European stock price dynamics. Second, in contrast to several works in the extant literature which assume the exogeneity of oil prices with respect to macroeconomic and financial variables, we document that a reverse relationship may exist, 
i.e., changes in some European sector returns do significantly affect world oil prices. These findings are consistent with the results established by some recent papers (Ewing and Thompson, 2007; Kilian and Vega, 2008; Lescaroux and Mignon, 2008).

Table 6. Results of the Granger causality tests

\begin{tabular}{|c|c|c|c|c|c|c|c|c|}
\hline Lags & 1 & 2 & 3 & 4 & 6 & 8 & 10 & 12 \\
\hline \multicolumn{9}{|c|}{ DJ Stoxx Europe } \\
\hline $\mathrm{S} \rightarrow \mathrm{O}$ & 0.073 & 0.075 & 0.111 & 0.099 & 0.216 & 0.112 & 0.104 & 0.362 \\
\hline $\mathrm{O} \rightarrow \mathrm{S}$ & 0.253 & 0.035 & 0.055 & 0.097 & 0.159 & 0.172 & 0.185 & 0.372 \\
\hline \multicolumn{9}{|c|}{ Automobile and Parts } \\
\hline $\mathrm{S} \rightarrow \mathrm{O}$ & 0.016 & 0.019 & 0.037 & 0.062 & 0.049 & 0.041 & 0.035 & 0.138 \\
\hline $\mathrm{O} \rightarrow \mathrm{S}$ & 0.133 & 0.007 & 0.007 & 0.006 & 0.005 & 0.011 & 0.023 & 0.048 \\
\hline \multicolumn{9}{|c|}{ Financials } \\
\hline $\mathrm{S} \rightarrow \mathrm{O}$ & 0.345 & 0.137 & 0.216 & 0.191 & 0.363 & 0.234 & 0.140 & 0.436 \\
\hline $\mathrm{O} \rightarrow \mathrm{S}$ & 0.137 & 0.076 & 0.117 & 0.155 & 0.277 & 0.077 & 0.150 & 0.272 \\
\hline \multicolumn{9}{|c|}{ Food and Beverages } \\
\hline $\mathrm{S} \rightarrow \mathrm{O}$ & 0.055 & 0.023 & 0.005 & 0.002 & 0.004 & 0.003 & 0.004 & 0.015 \\
\hline $\mathrm{O} \rightarrow \mathrm{S}$ & 0.102 & 0.064 & 0.150 & 0.086 & 0.119 & 0.142 & 0.178 & 0.315 \\
\hline \multicolumn{9}{|c|}{ Oil and Gas } \\
\hline $\mathrm{S} \rightarrow \mathrm{O}$ & 0.068 & 0.060 & 0.058 & 0.069 & 0.056 & 0.082 & 0.063 & 0.160 \\
\hline $\mathrm{O} \rightarrow \mathrm{S}$ & 0.635 & 0.040 & 0.136 & 0.144 & 0.306 & 0.341 & 0.470 & 0.487 \\
\hline \multicolumn{9}{|c|}{ Health Care } \\
\hline $\mathrm{S} \rightarrow \mathrm{O}$ & 0.632 & 0.441 & 0.250 & 0.324 & 0.456 & 0.158 & 0.161 & 0.468 \\
\hline $\mathrm{O} \rightarrow \mathrm{S}$ & 0.128 & 0.055 & 0.149 & 0.272 & 0.510 & 0.499 & 0.377 & 0.445 \\
\hline \multicolumn{9}{|c|}{ Industrials } \\
\hline $\mathrm{S} \rightarrow \mathrm{O}$ & 0.030 & 0.022 & 0.043 & 0.025 & 0.060 & 0.032 & 0.039 & 0.180 \\
\hline $\mathrm{O} \rightarrow \mathrm{S}$ & 0.807 & 0.057 & 0.063 & 0.106 & 0.154 & 0.087 & 0.072 & 0.166 \\
\hline \multicolumn{9}{|c|}{ Basic Materials } \\
\hline $\mathrm{S} \rightarrow \mathrm{O}$ & 0.005 & 0.004 & 0.009 & 0.007 & 0.003 & 0.001 & 0.001 & 0.008 \\
\hline $\mathrm{O} \rightarrow \mathrm{S}$ & 0.625 & 0.264 & 0.426 & 0.195 & 0.338 & 0.251 & 0.280 & 0.404 \\
\hline \multicolumn{9}{|c|}{ Personal and Household Goods } \\
\hline $\mathrm{S} \rightarrow \mathrm{O}$ & 0.070 & 0.042 & 0.074 & 0.085 & 0.163 & 0.078 & 0.060 & 0.246 \\
\hline $\mathrm{O} \rightarrow \mathrm{S}$ & 0.069 & 0.097 & 0.144 & 0.259 & 0.343 & 0.566 & 0.308 & 0.451 \\
\hline \multicolumn{9}{|c|}{ Consumer Services } \\
\hline$S \rightarrow O$ & 0.056 & 0.038 & 0.063 & 0.116 & 0.308 & 0.155 & 0.127 & 0.303 \\
\hline $\mathrm{O} \rightarrow \mathrm{S}$ & 0.131 & 0.069 & 0.090 & 0.154 & 0.184 & 0.247 & 0.141 & 0.285 \\
\hline \multicolumn{9}{|c|}{ Technology } \\
\hline$S \rightarrow O$ & 0.529 & 0.803 & 0.903 & 0.400 & 0.454 & 0.469 & 0.532 & 0.852 \\
\hline $\mathrm{O} \rightarrow \mathrm{S}$ & 0.324 & 0.084 & 0.112 & 0.208 & 0.112 & 0.016 & 0.005 & 0.020 \\
\hline \multicolumn{9}{|c|}{ Telecommunications } \\
\hline $\mathrm{S} \rightarrow \mathrm{O}$ & 0.582 & 0.811 & 0.902 & 0.606 & 0.791 & 0.807 & 0.771 & 0.909 \\
\hline $\mathrm{O} \rightarrow \mathrm{S}$ & 0.607 & 0.116 & 0.222 & 0.233 & 0.196 & 0.213 & 0.149 & 0.247 \\
\hline \multicolumn{9}{|c|}{ Utilities } \\
\hline $\mathrm{S} \rightarrow \mathrm{O}$ & 0.046 & 0.091 & 0.138 & 0.240 & 0.393 & 0.387 & 0.313 & 0.531 \\
\hline $\mathrm{O} \rightarrow \mathrm{S}$ & 0.673 & 0.087 & 0.212 & 0.322 & 0.597 & 0.768 & 0.871 & 0.943 \\
\hline
\end{tabular}

Notes: The Granger tests are based on a linear VAR(P) model, where $p$ is equal to $1,2,3,4,6,8,10$ and 12, respectively.The table provides the $p$-values of rejection of the null hypothesis.

$\mathrm{S} \rightarrow \mathrm{O}$ is the null hypothesis of no Granger causality from stock market returns to oil price changes.

$\mathrm{O} \rightarrow \mathrm{S}$ is the null hypothesis of no Granger causality from oil price changes to stock market returns.

\subsection{Cyclical correlations between oil and stock markets}

To the extent that variations in macroeconomic fundamentals may influence the direct shortrun linkages between oil price changes and sector stock returns in Europe, it is relevant to investigate the cyclical correlations between variables of interest. To do so, we follow the methodology introduced by Serletis and Shahmoradi (2005) and applied by several papers to study the links between energy prices and economics activity (Ewing and Thompson, 2007; Lescaroux and Mignon, 2008). First, the Hodrick-Prescott (HP) filter is employed to 
decompose each time-series variable in our study into long-run and business cycle components. We then compute the cross-correlations between the cyclical component of oil price $\left(\right.$ coil $\left._{t}\right)$ and that of sector stock market indices $\left(\right.$ cstock $\left._{t}\right)$. We denote these correlations by $\rho(j)$ and they are computed for $j=0, \pm 1, \pm 2, \pm 3, \pm 4, \pm 5$ and \pm 6 . Therefore, the cyclical correlations permit to assess the linkages that may exist between oil price and stock markets over the business cycle. They enable, in particular, the investigation of the dynamics of the short-run component comovements by providing information about both their strength and their synchronization. Following Serletis and Shahmoradi (2005), and Ewing and Thompson (2007), we consider that the two cyclical components are strongly correlated, weakly correlated, or uncorrelated for a shift $j$ based on $0.23 \leq|\rho(j)|<1,0.10 \leq|\rho(j)|<0.23,0 \leq|\rho(j)|$ $<0.10$, respectively. If $|\rho(j)|$ is high for a positive, zero, or negative value of $j$, then the cycle of oil prices is leading the cycle of stock markets by $j$ periods, is synchronous, or is lagging behind the cycle of stock markets by $j$ periods, respectively.

Table 7. Cyclical correlations of oil prices with stock market indices

\begin{tabular}{|c|c|c|c|c|c|c|c|c|c|c|c|c|c|}
\hline Period $j$ & -6 & -5 & -4 & -3 & -2 & -1 & 0 & 1 & 2 & 3 & 4 & 5 & 6 \\
\hline $\begin{array}{l}\text { DJ Stoxx } \\
\text { Europe }\end{array}$ & 0.024 & 0.051 & 0.087 & 0.107 & 0.119 & 0.118 & 0.116 & 0.070 & 0.023 & -0.011 & -0.042 & -0.080 & -0.907 \\
\hline $\begin{array}{l}\text { Automobile } \\
\text { and Parts }\end{array}$ & 0.020 & 0.026 & 0.046 & 0.008 & 0.023 & -0.064 & -0.000 & -0.170 & -0.200 & -0.204 & -0.243 & -0.257 & -0.282 \\
\hline Financials & -0.073 & -0.066 & -0.044 & -0.035 & -0.025 & -0.031 & -0.187 & -0.202 & -0.206 & -0.196 & -0.183 & -0.181 & -0.169 \\
\hline $\begin{array}{l}\text { Food and } \\
\text { Beverages }\end{array}$ & 0.023 & -0.012 & -0.045 & -0.074 & -0.093 & -0.127 & -0.158 & -0.166 & -0.164 & -0.169 & -0.256 & -0.161 & -0.167 \\
\hline Oil and Gas & 0.281 & 0.301 & 0.330 & 0.356 & 0.382 & 0.352 & 0.385 & 0.320 & 0.271 & 0.231 & 0.196 & 0.151 & 0.105 \\
\hline Health Care & -0.193 & -0.202 & -0.203 & -0.294 & -0.191 & -0.194 & -0.183 & -0.153 & -0.128 & -0.101 & -0.073 & -0.055 & -0.036 \\
\hline Industrials & 0.096 & 0.088 & 0.089 & 0.079 & 0.069 & 0.043 & 0.016 & -0.014 & -0.041 & -0.054 & -0.069 & -0.090 & -0.108 \\
\hline Basic Materials & 0.422 & 0.430 & 0.455 & 0.465 & 0.452 & 0.423 & 0.422 & 0.360 & 0.304 & 0.263 & 0.222 & 0.180 & 0.131 \\
\hline $\begin{array}{l}\text { Personal and } \\
\text { Household Goods }\end{array}$ & 0.032 & 0.014 & -0.001 & -0.025 & -0.048 & -0.086 & -0.120 & -0.155 & -0.151 & -0.157 & -0.162 & -0.173 & -0.178 \\
\hline Consumer Services & -0.086 & -0.097 & -0.100 & -0.108 & -0.118 & -0.138 & -0.164 & -0.182 & -0.195 & -0.200 & -0.207 & -0.216 & -0.217 \\
\hline Technology & 0.038 & 0.034 & 0.037 & 0.035 & 0.036 & 0.036 & 0.033 & 0.026 & 0.018 & 0.012 & 0.005 & 0.000 & 0.005 \\
\hline $\begin{array}{l}\text { Telecom- } \\
\text { munications }\end{array}$ & -0.020 & -0.021 & -0.024 & -0.030 & -0.031 & -0.031 & -0.034 & -0.039 & -0.046 & -0.050 & -0.052 & -0.052 & -0.049 \\
\hline Utilities & 0.002 & 0.001 & 0.011 & 0.016 & 0.024 & 0.025 & 0.011 & 0.002 & -0.024 & -0.033 & -0.040 & -0.065 & -0.095 \\
\hline
\end{tabular}

Notes: this table provides the cyclical correlations between oil price and stock market prices measured by $\rho(j)=\rho\left(\right.$ coil $_{t}$, cstock $\left._{t+j}\right)$. Bold type indicates high absolute value correlations.

The results for leads and lags from 1 to 6 are shown in Table 7. They globally confirm previous results. Positive weak-cyclical correlations are observed for the DJ Stoxx 600 Index 
for negative values of $j$, suggesting that oil prices are pro-cyclical and lagging the European stock market generally after few weeks over the last decade. As we expect, oil prices and stock markets are strongly and contemporaneously correlated for Oil and Gas, and Basic Materials industries. Furthermore, for two sectors (Automobile and Parts, and Food and Beverages) highly negative cyclical correlations are observed for positive values of $j$, indicating that oil prices are counter-cyclical and leading the stock markets generally by few weeks ahead in these sectors. Weak correlations are obtained for four sectors: Health Care, Industrials, Personal Goods and Consumer Services. Surprisingly, we find no significant cyclical correlations between oil prices and stock market returns in Technology and Utilities industries, but as expected no correlation is also found for Telecommunications stocks.

Overall, our analysis shows significant relationships between most sector returns in Europe and oil price changes. However, there is some asymmetry in the reaction of some sectors to oil price shocks, and in all cases the value and sign of the sensitivities of stock returns to oil price changes vary significantly across sectors. In what follows, we carry out some robustness checks.

\subsection{Robustness checks}

To check for the robustness of our empirical results, the following changes are made. First, given previous evidence in the literature on the significance of world market risk and exchange rate risk within international asset pricing model settings, especially in developed stock markets such as European markets, we re-estimate our empirical models in the presence of the world market returns and the euro-dollar exchange rate changes. Second, we reestimate our models using different sample periods and data frequencies. Finally, our empirical results are re-examined using real oil and stock prices instead of nominal prices in order to take into account the effects of inflation. ${ }^{8}$

\section{a) Effects of exchange rates}

Oil is commonly priced in US dollars in world oil markets, and thus the latter play an important role in the energy industry to the extent that the dollar exchange rate affects directly the price at which producers and consumers perceive oil and oil-related products. Therefore, it is interesting to consider the potential links between the dollar exchange rates and the oil prices in our empirical investigation. In the literature, the link between the dollar and oil prices has been examined at theoretical levels by, among others, Krugman (1980) and Rogoff (1991), and at empirical levels by, among others, Zhou (1995), and Dibooglu (1995). The

\footnotetext{
${ }^{8}$ In order to preserve space, the results of robustness tests are not reported here, but available upon request.
} 
findings of these studies suggest the existence of a positive relationship between the two variables, i.e., a rise in oil prices is linked to a dollar appreciation.

Moreover, recent papers show that exchange rate risk is internationally priced (De Santis et $a l ., 2003$ and references therein). In this schema of things, the statistical significance of oil price changes in Models 2 and 3 could be due to the failure to include the euro-dollar exchange rate changes. To shed light on this issue, we re-estimate Models 2 and 3 in presence of the euro-dollar exchange rate changes as a risk factor. The results indicate that changes in exchange rate have significant effects on most European sector returns. Meanwhile, the relationships between oil price shocks and European sector returns remain mainly unchanged: oil price changes positively affect sector returns in five cases (Financials, Oil and Gas, Industrials, Basic Materials, and Consumer Services) and negatively in four cases (Automobile and Parts, Food and Beverages, Health Care and Technology). Strong signs of asymmetric reaction to oil price changes have been found, in particular for Food and Beverages, and Personal and Household Goods stock returns.

\section{b) Effects of world stock market}

If European markets are integrated into world stock market, it is reasonable to believe that European stock returns are exposed to the world market risk. This leads us to test for the significance of oil price changes within an asset pricing model that allows for world market risk to affect stock returns as a robustness check. The inclusion of this global factor is motivated by the fact that if oil price changes and world market returns are correlated, tests based on models as in Equations (2) and (3) may result in a spurious significance of the oil price changes because of the failure to account for global factors.

More precisely, we re-estimate augmented versions of Models 2 and 3 which incorporate the part of MSCI world market returns, unexplained by the DJ Stoxx index returns and Brent oil price changes. We find that the global factor, independent from European and oil factors are significant for several European sectors. However, the significance of the oil price changes seems to be unaffected after the introduction of the global factor.

\section{c) Alternative sample periods and frequencies}

The DJ Stoxx sector indices are launched in 1998, but their daily, weekly and monthly frequencies were built back to December 31, 1986. As an exercise of the robustness check, we test the sensitivity of our results to the choice of the sample periods and data frequency. Concretely, we re-estimate our different models using two different data frequencies (daily and monthly) as well as a longer sample period from 1986 onwards. 
The evidence globally indicates that our previous conclusions on the oil-stock return relationships hold for a longer sample period and regardless of data frequencies used. It means that empirical results we report in the paper are reasonably robust. The main differences we should mention are the following: $i$ ) when using monthly data, we find that the ARCH effects are slightly weaker and that the monthly frequency displays some bearing on asymmetry. $i$ ) when daily data are used, AR(1) coefficients appear to be significant in higher number of cases, compared to weekly data.

\section{d) Real versus nominal prices}

The last step of our robustness check consists of examining whether our results subject to changes or not with respect to the effects of inflation. For this we repeat the same empirical analysis as above using real oil and stock prices instead of nominal prices. Globally, we realize that our results do not change significantly and our main conclusions remain valid, i.e., oil price changes positively affect sector returns in five cases (Financials, Oil and Gas, Industrials, Basic Materials, and Consumer Services) and negatively in three cases (Food and Beverages, Health Care and Technology). Weak negative relationships have been found for Automobile and Parts and Personal and Household Goods stock returns.

In summary, the evidence discussed in this section suggests that oil price changes significantly affect several European sectors, even after European market, world market and exchange rate risk factors as well as inflation effects are accounted for. These findings are also robust to the choice of the sample period and the frequency of data.

\section{Some portfolio implications of the results}

In this section, we discuss some implications of the results we obtain for portfolio investment. First, we show that a model with oil risk presents superior out-of-sample forecasting results to a market model. Second, we illustrate how our results can be used in portfolio diversification and measure the out-of-sample benefit from portfolios considering these results. Note that we report, in what follows, the results obtained with weekly data over the out-of-sample subperiod running from November 20, 2008 to December 31, 2009 which totalizes 59 observations. $^{9}$

\subsection{Out-of-sample forecasts of sector stock market returns}

Among the many issues involving portfolio investment and management, modelling and forecasting stock returns are one of the most intriguing topics that attract great interests from

\footnotetext{
${ }^{9}$ The authors are very grateful to the anonymous referee and to Stéphane Grégoir for very interesting comments and valuable suggestions that have greatly motivated this part of the paper.
} 
investors and researchers. Our analysis of causality and cycle synchronicity shows significant interactions between oil and sector stock returns as well as some predictability in stock price dynamics based on oil price changes. Moreover, as we have pointed out in sub-section 3.1, Models 2 and 3 provide better fit to our data than the market model (Model 1) in most cases. However, this does not guarantee that models with symmetric and asymmetric responses of stock returns to oil price changes will perform better in actual forecasting of stock returns.

Recall that the aim of forecasting evaluation is to minimize the expected loss, i.e. the difference between the predicted and actual returns. There is, up to date, a wide range of standard statistical loss functions that can be used to evaluate such a deviation in forecasting tasks. In this paper, we retain the most commonly used loss functions, namely Root Mean Squared Error (RMSE), Mean Absolute Error (MAE), and Mean of Absolute Percent Error (MAPE). They are defined as

$$
\begin{aligned}
& R M S E_{i}=\sqrt{h^{-1} \sum_{t=T+1}^{T+h}\left(r_{i t}-\hat{r}_{i t}\right)^{2}} \\
& M A E_{i}=h^{-1} \sum_{t=T+1}^{T+h}\left|r_{i t}-\hat{r}_{i t}\right| \\
& M A P E_{i}=h^{-1} \sum_{t=T+1}^{T+h}\left|\frac{r_{i t}-\hat{r}_{i t}}{r_{i t}}\right|
\end{aligned}
$$

where $t$ denotes time period of the forecast sample, $t=T+1, T+2, \ldots, T+h . r_{i t}$ and $\hat{r}_{i t}$ stand

\begin{tabular}{|c|c|c|c|c|c|c|c|c|c|c|}
\hline \multirow{2}{*}{ Sectors } & \multirow{2}{*}{ Distribution } & \multicolumn{3}{|c|}{ Model 1} & \multicolumn{3}{|c|}{ Model 2} & \multicolumn{3}{|c|}{ Model 3} \\
\hline & & RMSE & MAE & MAPE & RMSE & MAE & MAPE & RMSE & MAE & MAPE \\
\hline \multirow{2}{*}{$\begin{array}{l}\text { Automobile and } \\
\text { Parts }\end{array}$} & Normal & 0.0353 & 0.0267 & 1.3141 & 0.0355 & 0.0270 & 1.2726 & 0.0356 & 0.0271 & 1.2764 \\
\hline & Student- $t$ & 0.0348 & 0.0265 & 1.2966 & 0.0353 & 0.0268 & 1.2617 & 0.0354 & 0.0268 & 1.2656 \\
\hline \multirow[t]{2}{*}{ Financials } & Normal & 0.0293 & 0.0205 & 0.7737 & 0.0286 & 0.0197 & 0.7765 & 0.0288 & 0.0199 & 0.7768 \\
\hline & Student- $t$ & 0.0292 & 0.0207 & 0.7765 & 0.0285 & 0.0194 & 0.7770 & 0.0290 & 0.0201 & 0.7783 \\
\hline \multirow{2}{*}{$\begin{array}{l}\text { Food and } \\
\text { Beverages }\end{array}$} & Normal & 0.0195 & 0.0145 & 1.5296 & 0.0186 & 0.0138 & 13797 & 0.0176 & 0.0133 & 14006 \\
\hline & Student- $t$ & 0.0195 & 0.0146 & 1.5465 & 0.0184 & 0.0137 & 13846 & 0.0172 & 0.0132 & 13831 \\
\hline \multirow[t]{2}{*}{ Oil and Gas } & Normal & 0.0195 & 0.0155 & 0.9817 & 0.0185 & 0.0136 & 1.2010 & 0.0189 & 0.0138 & 1.2173 \\
\hline & Student- $t$ & 0.0193 & 0.0154 & 0.9905 & 0.0184 & 0.0133 & 1.2169 & 0.0188 & 0.0138 & 1.2133 \\
\hline \multirow[t]{2}{*}{ Health Care } & Normal & 0.0207 & 0.0155 & 1.8177 & 0.0198 & 0.0147 & 1.3644 & 0.0188 & 0.0144 & 1.3595 \\
\hline & Student- $t$ & 0.0207 & 0.0152 & 1.7528 & 0.0195 & 0.0142 & 1.4761 & 0.0191 & 0.0141 & 1.5023 \\
\hline \multirow[t]{2}{*}{ Industrials } & Normal & 0.0147 & 0.0114 & 6.5534 & 0.0143 & 0.0090 & 6.5283 & 0.0148 & 0.0100 & 6.5977 \\
\hline & Student- $t$ & 0.0148 & 0.0116 & 6.6542 & 0.0142 & 0.0091 & 6.6051 & 0.0145 & 0.0102 & 6.5091 \\
\hline \multirow[t]{2}{*}{ Basic Materials } & Normal & 0.0234 & 0.0195 & 0.8726 & 0.0216 & 0.0178 & 0.8546 & 0.0212 & 0.0186 & 0.8690 \\
\hline & Student- $t$ & 0.0223 & 0.0186 & 0.8955 & 0.0224 & 0.0186 & 0.8463 & 0.0222 & 0.0180 & 0.8607 \\
\hline Personal \& & Normal & 0.0136 & 0.0095 & 1.8676 & 0.0121 & 0.0088 & 0.9875 & 0.0115 & 0.0076 & 0.9581 \\
\hline
\end{tabular}
for the actual and forecasted returns respectively.

Table 8. Forecasting evaluation results 


\begin{tabular}{|c|c|c|c|c|c|c|c|c|c|c|}
\hline $\begin{array}{l}\text { Household } \\
\text { Goods }\end{array}$ & Student- $t$ & 0.0128 & 0.0083 & 1.1230 & 0.0119 & 0.0089 & 0.9981 & 0.0117 & 0.0080 & 0.9463 \\
\hline \multirow{2}{*}{$\begin{array}{l}\text { Consumer } \\
\text { Services }\end{array}$} & Normal & 0.0145 & 0.0116 & 1.1535 & 0.0136 & 0.0113 & 1.0934 & 0.0139 & 0.0115 & 1.1098 \\
\hline & Student- $t$ & 0.0149 & 0.0111 & 1.1614 & 0.0133 & 0.0118 & 1.1071 & 0.0139 & 0.0117 & 1.0964 \\
\hline \multirow[t]{2}{*}{ Technology } & Normal & 0.0285 & 0.0200 & 4.2735 & 0.0287 & 0.0205 & 4.0283 & 0.0274 & 0.0206 & 3.6816 \\
\hline & Student- $t$ & 0.0272 & 0.0195 & 4.0336 & 0.0277 & 0.0204 & 2.6684 & 0.0286 & 0.0204 & 3.8187 \\
\hline \multirow{2}{*}{$\begin{array}{l}\text { Telecommunica- } \\
\text { tions }\end{array}$} & Normal & 0.0251 & 0.0177 & 5.4556 & 0.0227 & 0.0184 & 4.0576 & 0.0229 & 0.0173 & 7.0124 \\
\hline & Student- $t$ & 0.0256 & 0.0175 & 6.9991 & 0.0221 & 0.0171 & 4.9122 & 0.0225 & 0.0172 & 8.8588 \\
\hline \multirow[t]{2}{*}{ Utilities } & Normal & 0.0204 & 0.0165 & 2.2235 & 0.0241 & 0.0180 & 1.7849 & 0.0209 & 0.0168 & 2.0726 \\
\hline & Student- $t$ & 0.0203 & 0.0163 & 2.2371 & 0.0220 & 0.0167 & 2.2030 & 0.0205 & 0.0164 & 2.2005 \\
\hline
\end{tabular}

Notes: Bold numbers denote the lowest error statistics. Results are obtained with weekly data over the out-ofsample sub-period going from 11/20/2008 to 12/31/2009 (59 observations).

In Table 8, we provide out-of-sample forecast results for the three competitive models (i.e., Models from 1 to 3 ). First, forecast evaluation functions show that the models estimated assuming the Student's t-distribution has in most cases the lowest losses and thus are superior to models assuming normal distribution. Second, Model 2 shows better forecasting results than Model 1 for the industries for which the in-sample estimations and evaluations indicate that oil price risk is significant. We thus conclude that augmenting the market model with oil price changes leads to better forecasting of sector stock returns in most cases. Finally, Model 3 shows superior results to Model 2 for the industries for which we have found significant asymmetries in the oil-stock returns relationships, i.e., Personal and Household Goods, Food and Beverages, Basic Materials, and Health Care. As a result, for these industries taking into account asymmetric effects conducts to better stock returns forecasting and thus to better portfolio investment decisions.

\subsection{Result implications for out-of-sample portfolio diversification}

The main findings of our in-sample analysis can be summarized as follows: on average, stock and oil markets are weakly correlated, the reactions of stock returns to oil price changes differ greatly depending on the activity sector, and finally there are some signs of asymmetric interactions between oil and stock returns. In order to illustrate the out-of-sample benefit from portfolio diversification considering our results, we have adopted some index-based investment strategies by constructing several portfolios composed of both stocks and oil with different allocation rates, and report the main results of our portfolio simulations in Table $9 .{ }^{10}$

\footnotetext{
${ }^{10}$ Note that alternatively one can invest in futures crude oil contracts. However, since the correlations between returns on spot oil prices, and on 1-month and 3-month futures contracts are very high over our sample period (respectively 0.92 and 0.94 ), we have found similar results using futures oil contract returns in our portfolio simulations instead of oil price returns.
} 
Table 9. Stock portfolio diversification through Brent crude oil

\begin{tabular}{|c|c|c|c|c|c|c|c|c|c|}
\hline & Mean & Std. dev. & $\begin{array}{l}\text { Sharpe } \\
\text { ratio }\end{array}$ & $\begin{array}{c}\text { Sharpe ratio } \\
\text { variation }(\%)\end{array}$ & $\begin{array}{c}\text { Sharpe ratio } \\
\text { variation }(\%)(1)\end{array}$ & Min. & Max. & Skew. & Kurt. \\
\hline \multicolumn{10}{|l|}{ Panel $A$} \\
\hline $100 \%$ PF I & 0.523 & 3.180 & 0.152 & -- & -- & -8.808 & 3.180 & -0.873 & 3.761 \\
\hline $\begin{array}{l}90 \% \text { PF I } \\
10 \% \text { Crude Oil }\end{array}$ & 0.618 & 3.143 & 0.184 & 21.053 & 21.053 & -8.558 & 6.605 & -0.730 & 3.445 \\
\hline $\begin{array}{l}80 \% \text { PF I } \\
20 \% \text { Crude Oil }\end{array}$ & 0.712 & 3.183 & 0.211 & 14.674 & 38.816 & -8.357 & 8.096 & -0.535 & 3.332 \\
\hline $\begin{array}{l}70 \% \text { PF I } \\
30 \% \text { Crude Oil }\end{array}$ & 0.807 & 3.298 & 0.233 & 10.427 & 53.289 & -8.131 & 9.586 & -0.326 & 3.455 \\
\hline $\begin{array}{l}60 \% \text { PF I } \\
40 \% \text { Crude Oil } \\
\end{array}$ & 0.902 & 3.480 & 0.248 & 6.438 & 63.158 & -7.905 & 11.077 & -0.138 & 3.734 \\
\hline \multicolumn{10}{|l|}{ Panel B } \\
\hline $100 \%$ PF II & 0.421 & 2.118 & 0.180 & -- & -- & -5.743 & 4.619 & -0.518 & 3.531 \\
\hline $\begin{array}{l}90 \% \text { PF II } \\
10 \% \text { Crude Oil }\end{array}$ & 0.526 & 2.101 & 0.232 & 28.889 & 28.889 & -4.902 & 5.192 & -0.129 & 3.211 \\
\hline $\begin{array}{l}80 \% \text { PF II } \\
20 \% \text { Crude Oil }\end{array}$ & 0.631 & 2.218 & 0.267 & 15.086 & 48.333 & -4.054 & 6.840 & 0.224 & 3.491 \\
\hline $\begin{array}{l}70 \% \text { PF II } \\
30 \% \text { Crude Oil }\end{array}$ & 0.736 & 2.451 & 0.284 & 6.367 & 57.778 & -4.051 & 8.487 & 0.419 & 4.100 \\
\hline $\begin{array}{l}60 \% \text { PF II } \\
40 \% \text { Crude Oil } \\
\end{array}$ & 0.841 & 2.770 & 0.298 & 4.930 & 65.556 & -4.882 & 10.135 & 0.474 & 4.600 \\
\hline \multicolumn{10}{|l|}{ Panel C } \\
\hline $100 \%$ PF III & 0.789 & 4.008 & 0.187 & -- & -- & -10.991 & 8.096 & -0.789 & 3.676 \\
\hline $\begin{array}{l}90 \% \text { PF III } \\
10 \% \text { Crude Oil }\end{array}$ & 0.875 & 3.902 & 0.214 & 14.439 & 14.439 & -10.547 & 9.288 & -0.623 & 3.553 \\
\hline $\begin{array}{l}80 \% \text { PF III } \\
20 \% \text { Crude Oil }\end{array}$ & 0.925 & 3.857 & 0.230 & 7.477 & 22.995 & -10.103 & 10.481 & -0.428 & 3.517 \\
\hline $\begin{array}{l}70 \% \text { PF III } \\
30 \% \text { Crude Oil }\end{array}$ & 0.994 & 3.876 & 0.247 & 7.391 & 32.086 & -9.659 & 11.673 & -0.230 & 3.608 \\
\hline $\begin{array}{l}60 \% \text { PF III } \\
40 \% \text { Crude Oil }\end{array}$ & 1.064 & 3.958 & 0.259 & 4.858 & 38.503 & -9.215 & 12.856 & -0.052 & 3.813 \\
\hline
\end{tabular}

Notes:

PF I is a weighted portfolio of all sectors, the DJ Stoxx portfolio;

PF II is an equally-weighted of sectors negatively linked to oil;

PF III is an equally-weighted of sectors positively linked to oil;

*: Sharpe ratio computed using the European 3-month interest rate;

(1): Variation in Sharpe ratio compared with a portfolio of $100 \%$ stock (PF I in Panel A, PF II in Panel B, and PF III in Panel C).

Results are obtained with weekly data over the out-of-sample sub-period going from 11/20/2008 to 12/31/2009 (59 observations).

In Panel A of Table 9, we quantify the benefit of introducing crude Brent oil into a diversified portfolio of all industries. We show that adding oil into the DJ Stoxx portfolio permits to enhance its risk-return characteristics. For instance, with only $10 \%$ of the portfolio invested in Brent crude oil, the average weekly return increases from $0.523 \%$ to $0.618 \%$, while the standard deviation decreases from $3.180 \%$ to $3.143 \%$. Consequently, the Sharpe ratio moves from 0.152 to 0.184 , and thus experiences an increase of more than $21 \%$. The skewness and kurtosis fall from -0.873 to -0.730 and from 3.761 to 3.445 respectively. These results correspond effectively to our expectations given the weak correlations between oil price changes and sector stock returns discussed in Sections 2 and 3. However, the marginal benefit from including crude oil into the DJ Stoxx portfolio is reduced all the more so the 
weight of crude oil in the combined portfolio increases as suggested by the Sharpe ratio's relative variations.

In Panels $\mathrm{B}$ and $\mathrm{C}$, we construct two equally-weighted portfolios based on the results of this paper. The portfolio PF II is an equally-weighted portfolio containing all industries negatively linked to oil price changes, while the portfolio PF III is an equally-weighted portfolio containing all industries positively related to oil price changes. The inspection of the results in Panels B and C show that the inclusion of crude oil in PF II and PF III leads to a significant reduction of the portfolio's standard deviations while ensuring a higher average return. Accordingly, the Sharpe ratio increases substantially in both cases. The diversification gain is however two times larger for portfolio PF II than for portfolio PF III, which is not surprising given the results we established in Section 3. For instance, when $10 \%$ of the portfolio is dedicated to investments in Brent crude oil, the average weekly return in panel B moves up from $0.421 \%$ to $0.526 \%$, and the standard deviation decreases from $2.118 \%$ to $2.101 \%$, leading the Sharpe ratio grows by about $29 \%$. At the same time, the investment risks associated with larger skewness and kurtosis fall as these coefficients diminish from -0.518 to only -0.129 and from 3.531 to 3.211 respectively. In Panel C, introducing $10 \%$ of oil into portfolio PF III leads to smaller improvements: the mean average increases from $0.789 \%$ to $0.875 \%$ and the standard deviation falls from $4.008 \%$ to $3.902 \%$, and thus the Sharpe ratio increases by only $14.439 \%$. The skewness and kurtosis decrease from -0.789 to only -0.623 and from 3.676 to 3.533 , respectively.

Overall, our portfolio simulations, based on the paper's results, lead to conclude that investors have interests to keep an eye on crude oil market movements in their investment decision-making process. Further, they can improve the risk-adjusted return of their portfolios by simply adopting a sector-based portfolio investment strategy on the basis of this paper's results.

\section{Conclusion}

In this article, we investigated the linkages between oil and stock prices. Unlike other empirical investigations, which have focused largely on broad market indices (national and/or regional indices), we contribute to a better understanding of the relationship between oil prices and the stock markets in Europe by testing for short-term links at both the aggregate and sector by sector levels. Our results show strong significant linkages between oil price changes and stock markets for most European sectors. However, the nature and sensitivity of the reaction of stock returns to oil price shocks change considerably across sectors. Our out- 
of-sample analysis reveals two important points. First, models with oil price changes as a factor of risk provide better forecasts of stock returns, and are thus more useful for portfolio investment decisions. Second, the inclusion of oil into a portfolio of stocks significantly improves its risk-return characteristics and a sector-based portfolio investment strategy considering the asymmetric responses of some industries to oil price shocks leads to a greater improvement of the Sharpe ratio.

Our findings should be of interest to researchers, regulators, and market participants. First, traders who are interested in investing in oil-sensitive stocks in Europe may, when oil prices are expected to increase, select stocks from sectors, such as Oil and Gas, with high positive sensitivity to oil prices. Alternatively, when oil price is expected to decrease, they may select sectors with negative sensitivity such as Food and Beverages. They can also use oil-related derivatives instruments. Thus, our results can be used to build profitable investment strategies. Second, the fact that sector returns in Europe have different sensitivities to oil price changes indicates valuable risk diversification opportunities across industries. Portfolio selection across rather than within sectors would be more efficient. Finally, investors and portfolio managers should rebalance their portfolios in keeping with their views of the sign of coming changes in oil prices (rises or falls), and our findings suggest that diversification can be achieved across sectors in all cases of oil price changes.

There are several avenues for future research. First, a sector analysis of the long-run linear and nonlinear links between oil and stock prices would be informative. Second, the econometric tools applied in this paper could be used to examine the effects of other energy products, such as natural gas. Third, a study of nonlinear causality between oil or other energy products and sector stock returns should be relevant. Finally, one of the future challenges would be to investigate whether oil price constitutes a common business cycle component across all European countries, that is affecting their sectoral indices indirectly. 


\section{References}

Apergis, N., Miller, S.M., 2009. Do structural oil-market shocks affect stock prices?. Energy Economics, 31, $569-575$.

Arouri M et Fouquau J, 2009, "On the Short-Term Influence of Oil Price Changes on Stock Markets in GCC Countries: Linear and Nonlinear Analyses ", Economics Bulletin, vol.29(2), 795-804.

Balaz, P., Londarev, A., 2006. Oil and its position in the process of globalization of the world economy. Politicka Ekonomie, 54, 508-528.

Bollerslev, T., Wooldrige, J.M. 1992. Quasi-maximum likelihood estimation and inference in dynamic models with time-varying covariances. Econometric Review, 11, 143-172.

Boyer, M.M., Filion, D., 2007. Common and fundamental factors in stock returns of Canadian oil and gas companies. Energy Economics, 29, 428-453.

Cologni, A., Manera, 2008. Oil prices, inflation and interest rates in a structural cointegrated VAR model for the G-7 countries. Energy Economics, 30, 856-888.

Cologni, A., Manera, M., 2009. The asymmetric effects of oil shocks on output growth: A markov-switching analysis for the G-7 countries. Economic Modelling, 26, 1-29.

Cunado, J., Perez de Garcia, F., 2005. Oil prices, economic activity and inflation: Evidence for some Asian countries. The Quarterly Review of Economics and Finance, 45, 65-83.

De Santis, G., Gerard, B., Hillion, P. 2003. The relevance of currency risk in the EMU. Journal of Economics and Business, 55, 427-462.

Dibooglu, S., 1995. Real disturbances, relative prices, and purchasing power parity. Southern Journal of Economics, 18, 69-87.

El-Sharif, I., Brown, D., Burton, B., Nixon, B., Russel, A., 2005. Evidence on the nature and extent of the relationship between oil and equity value in UK. Energy Economics, 27, 819-830.

Ewing, B.T., Thompson, M.A., 2007. Dynamic cyclical components of oil prices with industrial production, consumer prices, unemployment and stock prices. Energy Policy, 35, 5535-5540.

Faff, R., Brailsford, T., 2000. A test of a two factor 'market and oil' pricing model. Pacific Accounting Review, $12,61-77$.

Gronwald, M., 2008. Large oil shocks and the US economy: infrequent incidents with large effects. Energy Journal, 29, 151-71.

Hamilton, J.D., 2003. What is an oil shock?. Journal of Econometrics, 113, 363-398.

Huang, R.D., Masulis, R.W., Stoll, H.R., 1996. Energy shocks and financial markets. Journal of Futures Markets, $16,1-27$.

Jones, C.M., Kaul, G., 1996. Oil and the stock markets. Journal of Finance, 51, 463-491.

Kilian, L., 2008. Exogenous oil supply shocks: how big are they and how much do they matter for the US economy?. Review of Economics and Statistics, 90, 216-240. 
Kilian, L., Vega, C., 2008. Do energy prices respond to U.S. macroeconomic news? A test of the hypothesis of predetermined energy prices. FRB International Finance Discussion Paper, n 957.

Krugman, P., 1980. Oil and the dollar. NBER Working Paper Series, n 554.

Lardic, S., Mignon, V., 2006. The impact of oil prices on GDP in European countries: an empirical investigation based on asymmetric cointegration. Energy Policy, 34, 3910-3915.

Lardic, S., Mignon, V., 2008. Oil prices and economic activity: an asymmetric cointegration approach. Energy Economics, 30, 847-855.

Lescaroux, F., Mignon, V., 2008. On the influence of oil prices on economic activity and other macroeconomic and financial variables. OPEC Energy Review, 32, 343-380.

Nandha, M., Brooks, R., 2009. Oil prices and transport sector returns: an international analysis. Review of Quantitative Finance and Accounting, 33, 393-409.

Nandha, M., Faff, R., 2008. Does oil move equity prices? A global view. Energy Economics, 30, 986-997.

Park, J., Ratti, R.A., 2008. Oil price shocks and stock markets in the US and 13 European countries. Energy Economics, 30, 2587-2608.

Rogoff, K., 1991. Oil, productivity, government spending and the real yen-dollar exchange rate. FRB of San Francisco Working Papers, $\mathrm{n}^{\circ}$ 91-06.

Sadorsky, P., 1999. Oil price shocks and stock market activity. Energy Economics, 2, 449-469.

Sadorsky, P., 2001. Risk factors in stock returns of Canadian oil and gas companies. Energy Economics, 23, 1728.

Serletis, A., Shahmoradi, A., 2005. Business cycles and natural gas prices. OPEC Review, 29, 75-84.

Zhang, D., 2008. Oil shock and economic growth in Japan: a nonlinear approach. Energy Economics, 30, 23742390

Zhou, S., 1995. The response of real exchange rates to various economic shocks. Southern Journal of Economics, 61, 936-954. 